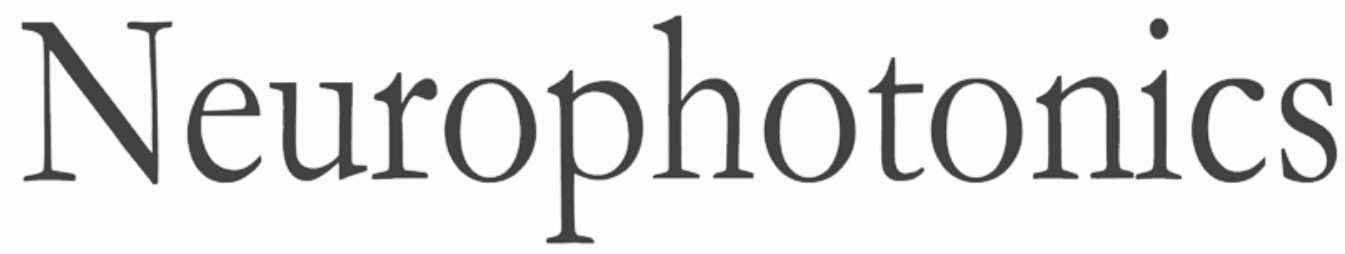

\title{
Denoising of neuronal signal from mixed systemic low-frequency oscillation using peripheral measurement as noise regressor in near-infrared imaging
}

Stephanie Sutoko

Yee Ling Chan

Akiko Obata

Hiroki Sato

Atsushi Maki

Takashi Numata

Tsukasa Funane

Hirokazu Atsumori

Masashi Kiguchi

Tong Boon Tang

Yingwei Li

Blaise deB Frederick

Yunjie Tong 


\title{
Denoising of neuronal signal from mixed systemic low-frequency oscillation using peripheral measurement as noise regressor in near-infrared imaging
}

\author{
Stephanie Sutoko, ${ }^{\mathrm{a}, *}$ Yee Ling Chan, ${ }^{\mathrm{b}}$ Akiko Obata, ${ }^{\mathrm{a}}$ Hiroki Sato, ${ }^{\mathrm{a}}$ Atsushi Maki, ${ }^{\text {a }}$ Takashi Numata, ${ }^{\mathrm{a}}$ \\ Tsukasa Funane, ${ }^{a}$ Hirokazu Atsumori, ${ }^{a}$ Masashi Kiguchi, ${ }^{a}$ Tong Boon Tang, ${ }^{b}$ Yingwei Li, ${ }^{\mathrm{c}, \mathrm{d}}$ \\ Blaise deB Frederick, ${ }^{\text {,e }}$ and Yunjie Tong ${ }^{\mathrm{a}, \mathrm{f}}$ \\ ${ }^{a}$ Hitachi, Ltd., Center for Exploratory Research, Research \& Development Group, Akanuma, Hatoyama, Saitama, Japan \\ 'Universiti Teknologi PETRONAS, Electrical and Electronic Engineering Department, Bandar Seri Iskandar, Tronoh Perak, Malaysia \\ 'McLean Hospital, Brain Imaging Center, Belmont, Massachusetts, United States \\ 'Yanshan University, School of Information Science and Engineering, Qinhuangdao, China \\ eHarvard Medical School, Department of Psychiatry, Boston, Massachusetts, United States \\ fPurdue University, Weldon School of Biomedical Engineering, West Lafayette, Indiana, United States
}

\begin{abstract}
Functional near-infrared spectroscopy (fNIRS) is a noninvasive functional imaging technique measuring hemodynamic changes including oxygenated $\left(\mathrm{O}_{2} \mathrm{Hb}\right)$ and deoxygenated $(\mathrm{HHb})$ hemoglobin. Low frequency (LF; 0.01 to $0.15 \mathrm{~Hz}$ ) band is commonly analyzed in fNIRS to represent neuronal activation. However, systemic physiological artifacts (i.e., nonneuronal) likely occur also in overlapping frequency bands. We measured peripheral photoplethysmogram (PPG) signal concurrently with fNIRS (at prefrontal region) to extract the low-frequency oscillations (LFOs) as systemic noise regressors. We investigated three main points in this study: (1) the relationship between prefrontal fNIRS and peripheral PPG signals; (2) the denoising potential using these peripheral LFOs, and (3) the innovative ways to avoid the false-positive result in fNIRS studies. We employed spatial working memory (WM) and control tasks (e.g., resting state) to illustrate these points. Our results showed: (1) correlation between signals from prefrontal fNIRS and peripheral PPG is region-dependent. The high correlation with peripheral ear signal (i.e., $\mathrm{O}_{2} \mathrm{Hb}$ ) occurred mainly in frontopolar regions in both spatial WM and control tasks. This may indicate the finding of task-dependent effect even in peripheral signals. We also found that the PPG recording at the ear has a high correlation with prefrontal fNIRS signal than the finger signals. (2) The systemic noise was reduced by $25 \%$ to $34 \%$ on average across regions, with a maximum of $39 \%$ to $58 \%$ in the highly correlated frontopolar region, by using these peripheral LFOs as noise regressors. (3) By performing the control tasks, we confirmed that the statistically significant activation was observed in the spatial WM task, not in the controls. This suggested that systemic (and any other) noises unlikely violated the major statistical inference. (4) Lastly, by denoising using the task-related signals, the significant activation of region-of-interest was still observed suggesting the manifest task-evoked response in the spatial WM task. () The Authors. Published by SPIE under a Creative Commons Attribution 3.0 Unported License. Distribution or reproduction of this work in whole or in part requires full attribution of the original publication, including its DOI. [DOI: 10.1117/1 .NPh.6.1.015001]
\end{abstract}

Keywords: low-frequency oscillation; brain; peripheral; near-infrared spectroscopy; systemic noise; denoising; working memory.

Paper 18028R received May 24, 2018; accepted for publication Dec. 10, 2018; published online Jan. 9, 2019.

\section{Introduction}

Functional near-infrared spectroscopy (fNIRS) is an imaging technique that noninvasively measures the product of cerebral hemodynamics (concentration changes of oxygenated and deoxygenated hemoglobin; $\Delta C_{\mathrm{O}_{2} \mathrm{Hb}}$ and $\Delta C_{\mathrm{HHb}}$ ) and optical path length $(L)$, using light in the near-infrared spectrum $(650$ to $900 \mathrm{~nm}){ }^{1-3}$ Because hemodynamic changes are related to local neuronal activity through neurovascular coupling, 4,5 fNIRS is commonly used to interpret brain activity and function. fNIRS has been widely used in research, clinical, and educational purposes ${ }^{6}$ due to its cost-effectiveness, safety, flexibility, higher spatial resolution than electroencephalography, and better temporal resolution than functional magnetic resonance

*Address all correspondence to Stephanie Sutoko, E-mail: stephanie.sutoko .tc@ hitachi.com imaging (fMRI). ${ }^{7}$ fNIRS systems are more compact than fMRI, which enables practical and continuous bedside monitoring even in infants and young children. ${ }^{8-10}$ fNIRS provides better motion tolerance; therefore, fNIRS is suitable for examining the challenging patients with restless symptoms ${ }^{11,12}$ and subjects actively engaging in movement such as walking and running. ${ }^{13,14}$ Furthermore, cochlear implant patients can safely undergo fNIRS measurement because there is no magnetic field that may endanger patients. ${ }^{15,16}$

Despite those advantages, there are three confounding factors in fNIRS studies. These factors are: (1) the mixture of neuronal and systemic physiological (nonneuronal) signals which is found in the low frequency (LF) range ${ }^{17,18}$ especially when the activation period is relatively short. ${ }^{19,20}$ This nonneuronal signal is compounded by several sources such as Mayer waves and vasomotion. ${ }^{21-25}$ In addition, because fNIRS data are measured through the intact skull, so every fNIRS channel 
has extracerebral noises (from skin, skull, and blood vessels on the surface of the brain). As a result, fNIRS is more susceptible to systemic noises. (2) Performing tasks likely incorporates not only targeted activation but also untargeted ones in the nearby regions. ${ }^{26,27}$ Since fNIRS has a low spatial resolution $\left(>\mathrm{cm}^{3}\right)$ with limited channels, and each channel covers a large area (i.e., low spatial resolution), it is prone to detect both targeted and untargeted activation within the same channel. (3) The mixture neuronal and systemic physiological signals in the LF band, coupled with small sample numbers may affect statistical inferences. $^{28}$ As a recent study pointed out, ${ }^{29}$ false-positive rates in many neuroimaging studies were largely inflated. Moreover, Hocke et al. ${ }^{30}$ demonstrated that some data processes, such as low-pass filtering, can also artificially inflate statistical power. In summary, obtaining accurate brain activation from fNIRS requires (1) careful experimental design with one or more built-in controls and (2) effective denoising methodologies used in both data acquisition and analyses.

Regarding the problem of an equivocal signal mixture, many efforts have been made to denoise fNIRS signal; we can classify these efforts into four approaches. First, the intracranial signal is particularly extracted by regressing the extracerebral effects using multidistance (MD) short-detector (S-D) separations. ${ }^{31,32}$ This approach measures blood-related changes in the superficial layer (e.g., scalp and skull) by using the S-D separation and successfully eliminates extracerebral noises, ${ }^{33,34}$ however, systemic noise still remains, as it is present not only in the superficial but also in the deep (i.e., cortices) layers. ${ }^{35,36}$ Second, many studies attempted the advanced computational analysis to isolate the systemic noises from brain signals. Yamada et al. ${ }^{37}$ proposed the separation between functional and systemic signals based on the assumption of the negative and positive relationship between $\mathrm{O}_{2} \mathrm{Hb}$ and $\mathrm{HHb}$, respectively. Prince et al. ${ }^{38}$ applied a model of systemic and cerebral activity components with the assumption of exact frequencies in the state space estimation techniques. This method is limited by noise-stimulus phaselocking, ${ }^{17,18}$ and simple component modeling likely underestimate the signal complexity. Third, data-driven methods such as principal and independent component analyses (PCA and ICA) have been introduced to decompose mixed signals into subsets of statistically uncorrelated and independent components (ICs), respectively. ${ }^{39-41}$ The empirical component ${ }^{42,43}$ can be selected by maximizing the correlation and covariance of ICs in the repeated stimulus frame. ${ }^{44}$ However, the problem of noise-stimulus phase-locking again influenced ICs selection and signal reconstruction. Therefore, the assumption of statistical independence among ICs could be biased against the characteristics of systemic noise in the event of brain activation. Finally, systemic physiological signals (e.g., respiration rate and arterial blood pressure) are measured using multimodalities to provide accurate noise regressors. ${ }^{45,46}$ However, the systemic noise regressor is restricted by the number of physiological signal recordings. ${ }^{25}$ In addition, the excessive multimodal measurement might burden subject's convenience.

In this study, we explored several ways to improve the accuracy of fNIRS experiments. We used the spatial working memory (WM) task as one of the cognitive measures. For experimental design, first, we incorporated the systematic control task (i.e., motor control) to avoid untargeted brain activation, which can arise from a synchronized movement associated with the spatial WM task. Second, we also incorporated the resting state task as a control to rule out false positives. Third, we developed a denoising method based on a general linear model (GLM) ${ }^{47,48}$ using the simultaneous peripheral photoplethysmographic (PPG) measurement (e.g., ear and finger) as systemic noise regressors. By directly recording the peripheral signals that we believe contained only the systemic noise, we avoided the error of noise modeling and do not require the questionable assumption of independent noise components. We chose the ear and finger to be the noise regressors for the following reasons. (1) Based on previous concurrent fMRI/fNIRS research, ${ }^{49}$ the $\mathrm{O}_{2} \mathrm{Hb}$ signal recorded from finger has broad and high correlations with blood oxygen level dependent fMRI signals in the brain. (2) We believe that, compared to the finger, the ear, which is much closer to the brain on the vascular path, should share more systemic fluctuations with the brain. (3) There are no confounding neuronal signals in these peripheral sites as mentioned above. (4) These locations are easy to measure by peripheral PPG device.

\section{Materials and Methods}

\subsection{Subjects}

Seventeen healthy adults ( 6 females, 11 males, age $=40.1 \pm$ 11.1 y.o.; mean $\pm \mathrm{SD}$, range $=24$ to 57 y.o.) participated in this study. Their handedness was assessed with the Edinburgh Handedness Inventory: ${ }^{50} 16$ subjects were right-handed. Data were obtained according to the standards of the internal review board of the Research and Development group, Hitachi, Ltd. All subjects received a detailed explanation of the measurements to be performed and provided the informed consent before participating in this experiment. Unexpected technical problems occurred, and the affected data (one and two samples for spatial $\mathrm{WM}$ and motor control tasks, respectively) were excluded in data analysis.

\subsection{Task Paradigms}

Each subject was measured in two sessions as shown in Fig. 1. Each session lasted for $15 \mathrm{~min}$. All tasks were designed using the Platform of Stimuli and Tasks software (Hitachi Ltd., Central Research Lab.) and presented on a monitor put in front of subjects. The first session involved 15 trials of spatial WM task and 10 trials of motor control task [Figs. 1(a) and 1(b)]. As in previous spatial WM studies, ${ }^{51}$ subjects were asked to encode the position of four red squares among displayed eight squares within $1.5 \mathrm{~s}$ (target stimulus) and to maintain the spatial information for $7 \mathrm{~s}$. During the memory maintenance period, subjects kept their sight on a fixation cross appearing on the black background screen. The monitor displayed a red square among eight squares (the probe stimulus) after the maintenance period. In addition to evoking WM, subjects were required to switch into decision-making mode to retrieve information and respond. ${ }^{52,53}$ Subjects needed to indicate whether the position of the red square in the probe stimulus was identical with the target stimulus [Fig. 1(a)] within 2 s. Since subjects gave responses by pressing buttons, we were aware of motor-evoked activation in the motor cortex. ${ }^{2,54}$ In order to estimate the impact of button pressing (motor) alone, a motor control task (with no information encoding) was given following the spatial WM task (still in session 1). The motor control was designed to always show all the red squares in the target stimulus and a fixation cross during the maintenance period. In the probe stimulus, we showed white squares on the left and red on the right (or vice versa) and asked 


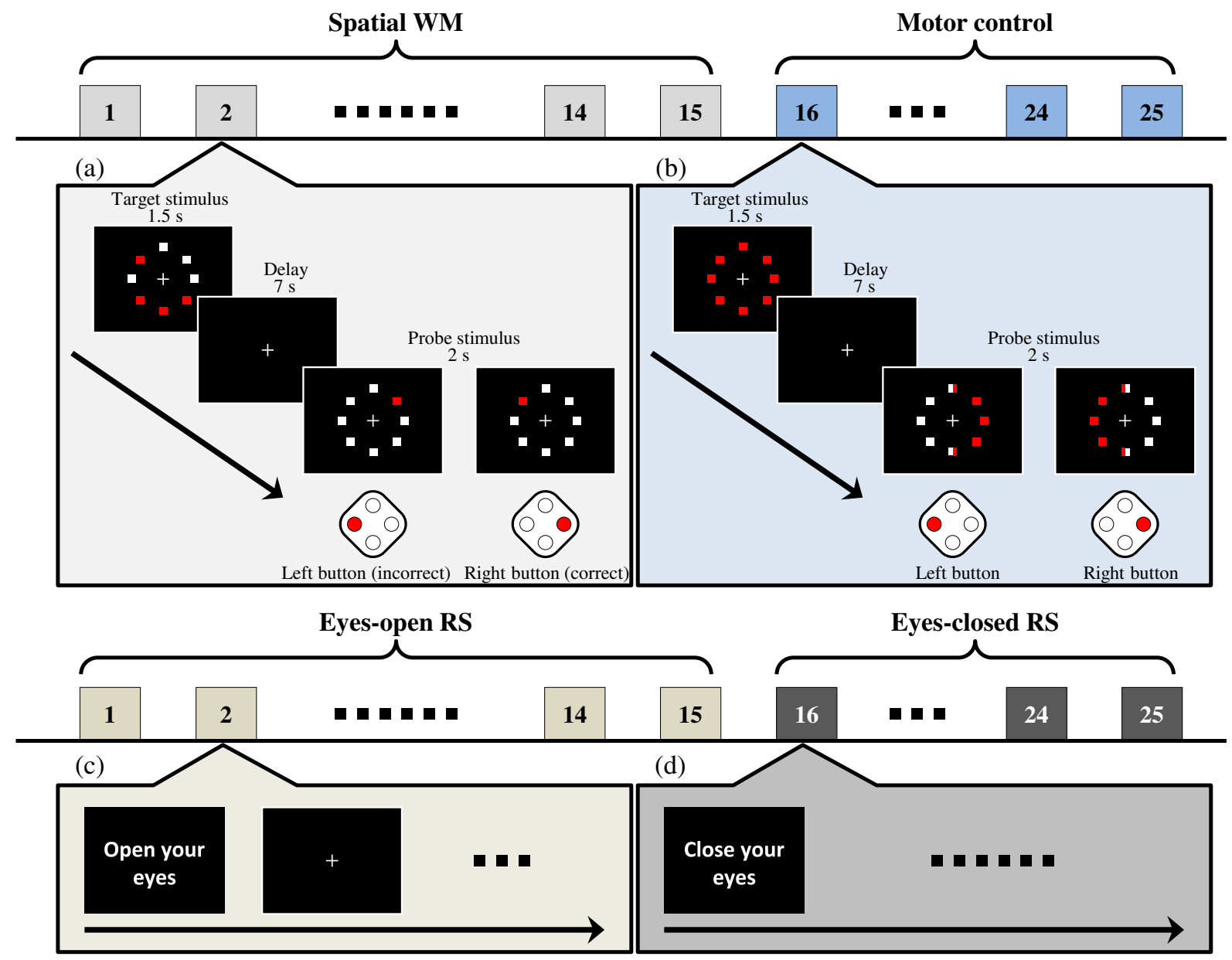

Fig. 1 Two measurement sessions (spatial WM, motor control; eyes-open, eyes-closed RS). Each session consisted of 25 trials; 15 trials for spatial WM/eyes-open RS and 10 trials for motor control/eyesclosed RS. (a) In the spatial WM task, subjects responded the probe stimulus by matching the position of red square in the target stimulus. (b) Meanwhile, the target stimulus was not required to be encoded and retrieved because subjects were merely required to answer which side majorly showed white squares during motor control. In the RS, subjects rested while (c) kept their eyes opened and (d) then closed their eyes.

the subjects to press button according to the location of the white squares [e.g., press left button if the white squares were on the left, see Fig. 1(b)]. The intertrial interval was randomized from 16 to $24 \mathrm{~s}$. In the second session, the resting state without any target and probe stimulus was used as another control task [Figs. 1(c) and 1(d)]. While resting, subjects fixed their sight on a fixation cross for the first 8 to 9 min [similar to the period for 15 trials; Fig. 1(c)] and closed their eyes for the remaining time [Fig. 1(d)]. The end of the second session was marked by soft beeping.

\subsection{Measurements}

Prefrontal measurements were acquired using an ETG-4000, a dual wavelength (695 and $830 \mathrm{~nm}$ ) fNIRS system (Hitachi Medical Corporation, Tokyo, Japan), with a sampling rate of $10 \mathrm{~Hz}$. One plane probe [Fig. 2(a), $3 \times 11 \mathrm{~S}$-D arrangement; 17 emitters and 16 detectors] with a 52-channel system was put on the subject's frontal lobe. The coordinates of the measured regions (i.e., channels) were estimated in the middle of each S-D pair and illustrated by the template of spatial registration [Fig. 2(b)]. ${ }^{55,56}$ Channels were categorized into four major regions based on the macroanatomy classification: bilateral postcentral, bilateral premotor, bilateral temporal, and frontopolar. The peripheral measurement was simultaneously conducted using multichannel PPG device (McLean Hospital, Massachusetts). This device was equipped with Nellcor type D-YS (ear-clip sensor) and DS-100A pulse oximeter (finger-clip sensor) probes with dual wavelengths (660 and $920 \mathrm{~nm}$ ) and sampling rate $31.25 \mathrm{~Hz} .{ }^{57}$ Ear-clip probes were attached to both ears and a finger-clip sensor was put on the left index finger [Fig. 2(c)].

\subsection{Data Preprocessing and Analysis}

Analyses were computed using MATLAB (The MathWorks, Inc.) and plug-in-based analysis platform, Platform for Optical Topography Analysis Tools (POTATo, developed by Hitachi Central Research Laboratory). ${ }^{58}$ Measured data from prefrontal and peripheral sites were both initially converted to the product of hemoglobin concentration changes and optical path length $(\Delta C \cdot L)$ for three signal types $\mathrm{O}_{2} \mathrm{Hb}, \mathrm{HHb}$, and $\mathrm{Hb}$-total following the modified Beer-Lambert equation. ${ }^{2,59}$ Figure 3(a) shows the flowchart of the analysis summary in which three analysis steps were performed. 
(a)
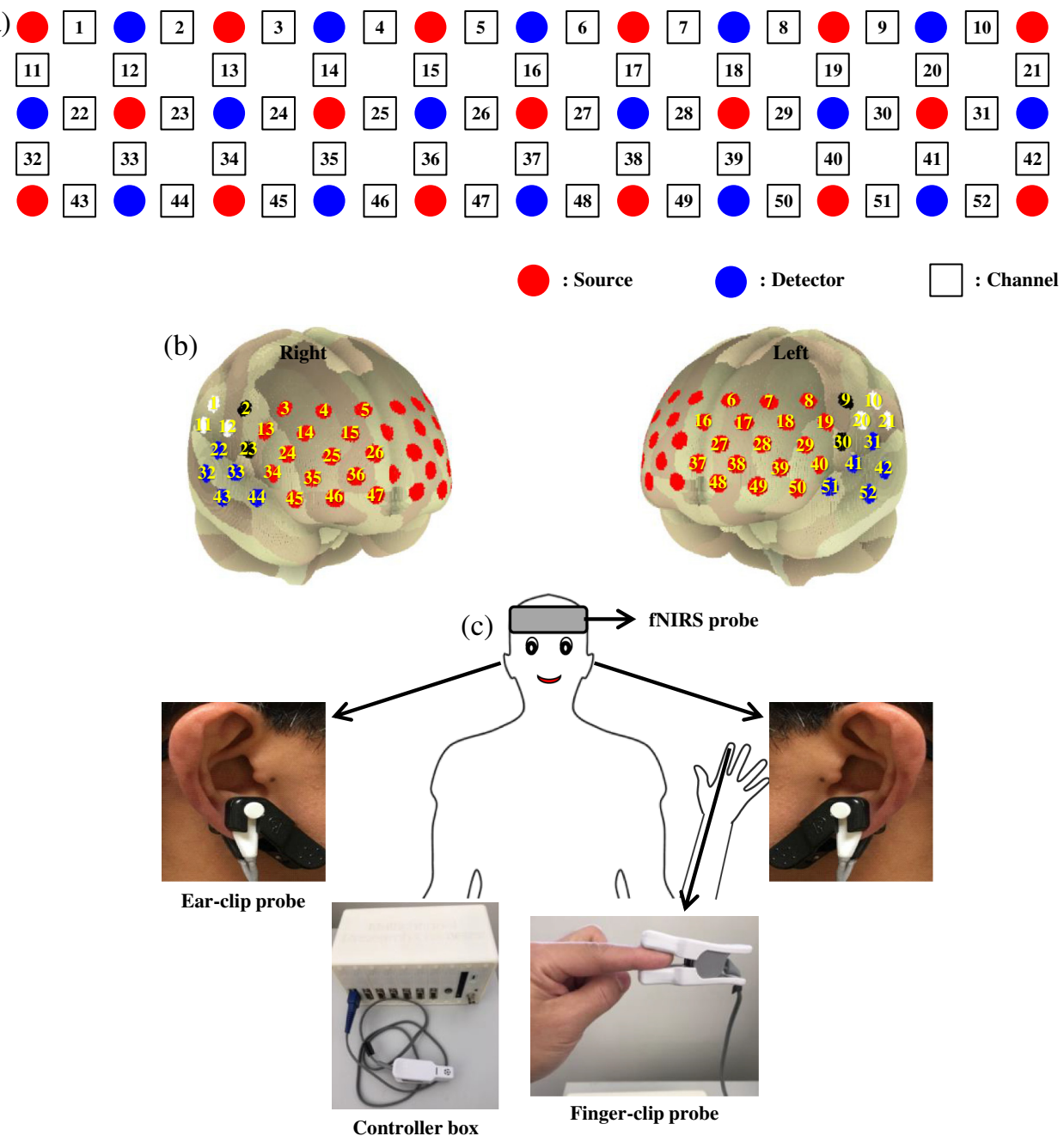

Fig. 2 (a) A plane of fNIRS probe holder with $3 \times 11$ configuration of source-detector (b) measured 52 channels that were spatially registered in frontal cortex. The frontal cortex was classified into four major channel regions: bilateral postcentral (i.e., white-colored channels), bilateral premotor (black-colored channels), bilateral temporal (blue-colored channels), and frontopolar (red-colored channels). The fNIRS probe was put on the subject's forehead whereas the PPG with controller box was connected to ear- and finger-clip probes fixed in subject's ears and left index finger.

\subsubsection{Processing without systemic denoising}

The continuous and channelwise fNIRS signal was bandpass filtered (0.01 to $0.15 \mathrm{~Hz}$; zero-phase Butterworth) ${ }^{51}$ The activation was estimated on the basis of the hemodynamic response function (HRF) in GLM analysis

$y=x \beta+\varepsilon$

where $y \in R^{L \times c h}$ ( $L=$ length of time points, ch = channel numbers) is fNIRS signals. $x \in R^{L \times N}$ is the regressor matrix $(N=$ number of regressor) designed by a constant, convolution between the boxcar function and HRF functions (i.e., canonical two-gamma, temporal and dispersion derivatives). The boxcar function corresponds to the time-piece of events (i.e., memory encoding and button pressing) as shown in Fig. 4(a). $\beta \in R^{N \times c h}$ is the estimated linear regressor of $x$ by the least square error, normally distributed $\varepsilon \in R^{L \times c h}$ with 0 mean and $\sigma^{2}$ variance: ${ }^{60}$ $h(t)=\frac{t^{\tau_{p}} e^{-t}}{\left(\tau_{p}\right) !}-\frac{t^{\left(\tau_{p}+\tau_{d}\right)} e^{-t}}{A\left(\tau_{p}+\tau_{d}\right) !}$,

where $h$ is the time $(t)$ function of canonical two-gamma HRF, ${ }^{61}$ $\tau_{p}$ is the parameter of first peak delay, $\tau_{d}$ is the parameter of second undershoot peak delay, and $A$ is the amplitude ratio between the first and second peaks. We used the typical parameters of 6,10 , and 6 for $\tau_{p}, \tau_{d}$, and $A$, respectively. Figure 4(b) shows the example of a regressor matrix. The model significance was statistically evaluated as follows:

$$
\begin{aligned}
& T_{\text {stat }}=\frac{c^{\prime} \beta}{\sqrt{\hat{\sigma}^{2} c^{\prime}\left(x^{\prime} x\right)^{-1} c}}, \\
& \hat{\sigma}^{2}=\frac{(y-x \beta)^{\prime}(y-x \beta)}{L-p},
\end{aligned}
$$

where $T_{\text {stat }}$ is the $t$-statistic value, $c$ is the contrast vector to infer

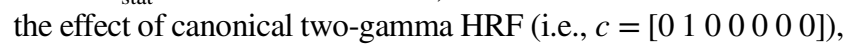


(a)

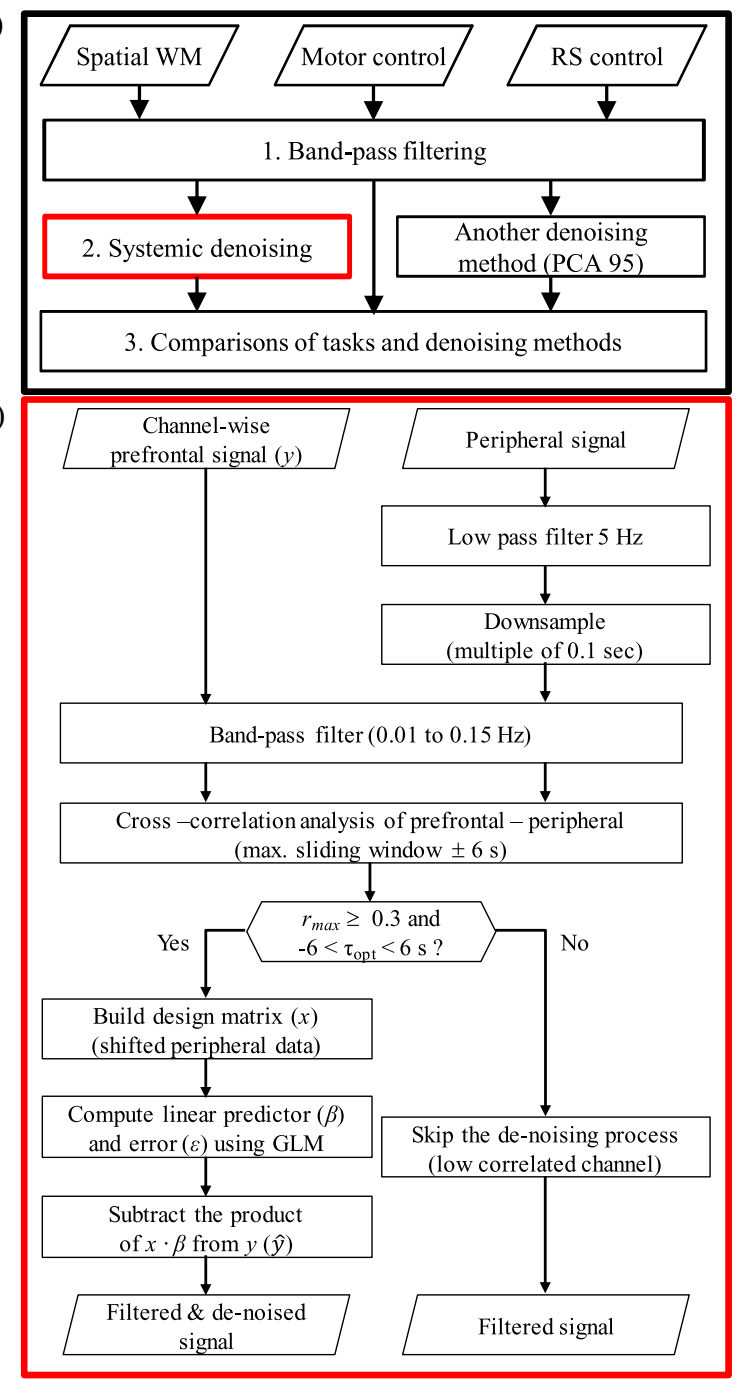

Fig. 3 (a) Overall summary of data analysis and (b) detail flow-chart for GLM-based systemic denoising process.

$\hat{\sigma}^{2}$ is the sum of squared error values corrected by $L-p$ degree of freedom $(p=\operatorname{rank} x){ }^{62-64}$ The channel significance was statistically evaluated in random effect group analyses using a onesample $t$-test of estimated $\beta$-values for the HRF regressor against zero. The strength of the phenomenon was assessed using the parameter of Cohen's $d$ effect size. The subject-level analysis evaluated the HRF $T_{\text {stat }}$ with $L-p$ degree of freedom.

\subsubsection{Processing with systemic denoising}

Systemic denoising was done for each task [Fig. 3(b)]. Therefore, after matching the sampling rate between fNIRS and peripheral PPG recordings, the continuous data were cut after the first 15 trials data, band-pass filtered in the LF window (0.01 to $0.15 \mathrm{~Hz}$; zero-phase Butterworth), and linearly detrended. The relationship between (channelwise) prefrontal fNIRS and peripheral PPG signals (i.e., $\mathrm{O}_{2} \mathrm{Hb}$ and $\mathrm{HHb}$ ) was assessed using the cross-correlation analysis. The maximum correlation $\left(r_{\max }\right)$ was determined within the confined sliding window $\pm 6 \mathrm{~s}$ based on the physiological characteristic of vascularization. ${ }^{49}$ The poor-quality signal may cause spurious correlations. According to a previous study, ${ }^{30}$ filtering and optimum delay search (i.e., cross correlation) elevate the correlation coefficient. In order to minimize the risk of spurious correlation, the threshold of $r_{\max }$ was set to be $0.3(p<0.01)$. The channelwise prefrontal signal having $r_{\max }$ less than 0.3 or greater than 0.3 with optimum delay $\left(\tau_{\mathrm{opt}}\right)$ at the boundary of sliding window (either -6 or 6 s) would left untouched. For denoising case, the peripheral signals would be shifted according to the delay (corresponding to the $r_{\max }$ ) to construct the systemic noise regressors. The use of dual peripheral regressors was also evaluated in which ear and finger signals were shifted independently. The systemic noise regressors were then combined with the typical $x$ regressor matrix (Fig. 4). Activation analysis was conducted following step 1 as $\beta$-values for the HRF regressor was also obtained while performing denoising. The signal reduction caused by the denoising process was evaluated to examine the portion of systemic noise in fNIRS signal by the following equations:

$$
\begin{aligned}
& \left.\mathrm{SNP}\right|_{c h}=\sqrt{\frac{\frac{1}{\Delta t} \int\left(y_{t, c h}-\hat{y}_{t, c h}\right)^{2} d t}{\frac{1}{\Delta t} \int\left(y_{t, c h}\right)^{2} d t}}, \\
& \hat{y}=y-x(I \beta)
\end{aligned}
$$

where SNP is the systemic noise portion, which is defined as the ratio between the square root of average signal difference caused by denoising $(y-\hat{y})$ and the raw signal $y$ in all time-point $t$ for each channel $c h, \hat{y}$ is the denoised signal, and $I \in R^{N \times N}$ is a square matrix in which diagonal elements associated with systemic noise and constant regressors are one. The efficiency of denoising was assessed through the change of activation significance and spatial distribution.

\subsubsection{Comparisons of tasks and denoising methods}

Both spatial WM and control (i.e., motor and resting state) tasks were analyzed in the same way as introduced in processing Sec. 2.4.1 without and Sec. 2.4 .2 with systemic denoising. In the motor control task, the result would interpret the activation pattern caused by button pressing, since no WM was involved. In the resting state task, the block design was invisible to subjects (only known by the computer). In our limited sample number, this resting state with no significant activation would assess the risk of the false-positive result. The comparison among tasks (e.g., peripheral correlation and signal reduction) was statistically done using the analysis of variance (ANOVA). In order to evaluate the current denoising feasibility, these datasets were also preprocessed by an existing method, PCA. ${ }^{43}$ PCA was performed independently for each signal type (i.e., $\mathrm{O}_{2} \mathrm{Hb}$ and $\mathrm{HHb}$ ). For component selection, PCA neglected components with total contribution less than $5 \%$.

\section{Results}

\subsection{Brain Activation in the Processing without Systemic Denoising}

We found the significantly increased $\mathrm{O}_{2} \mathrm{Hb}$ activations ( $p<0.05$; Holm-Bonferroni corrected) only in the spatial WM task but not in any control tasks [Figs. 5(a)-5(d)]. For the spatial WM task, the increase of $\mathrm{O}_{2} \mathrm{Hb}$ activation was significantly seen in both middle frontal (Broadmann area/BA 10, 45,46 , and 11) and bilateral temporal supramarginal areas, with 
(a)
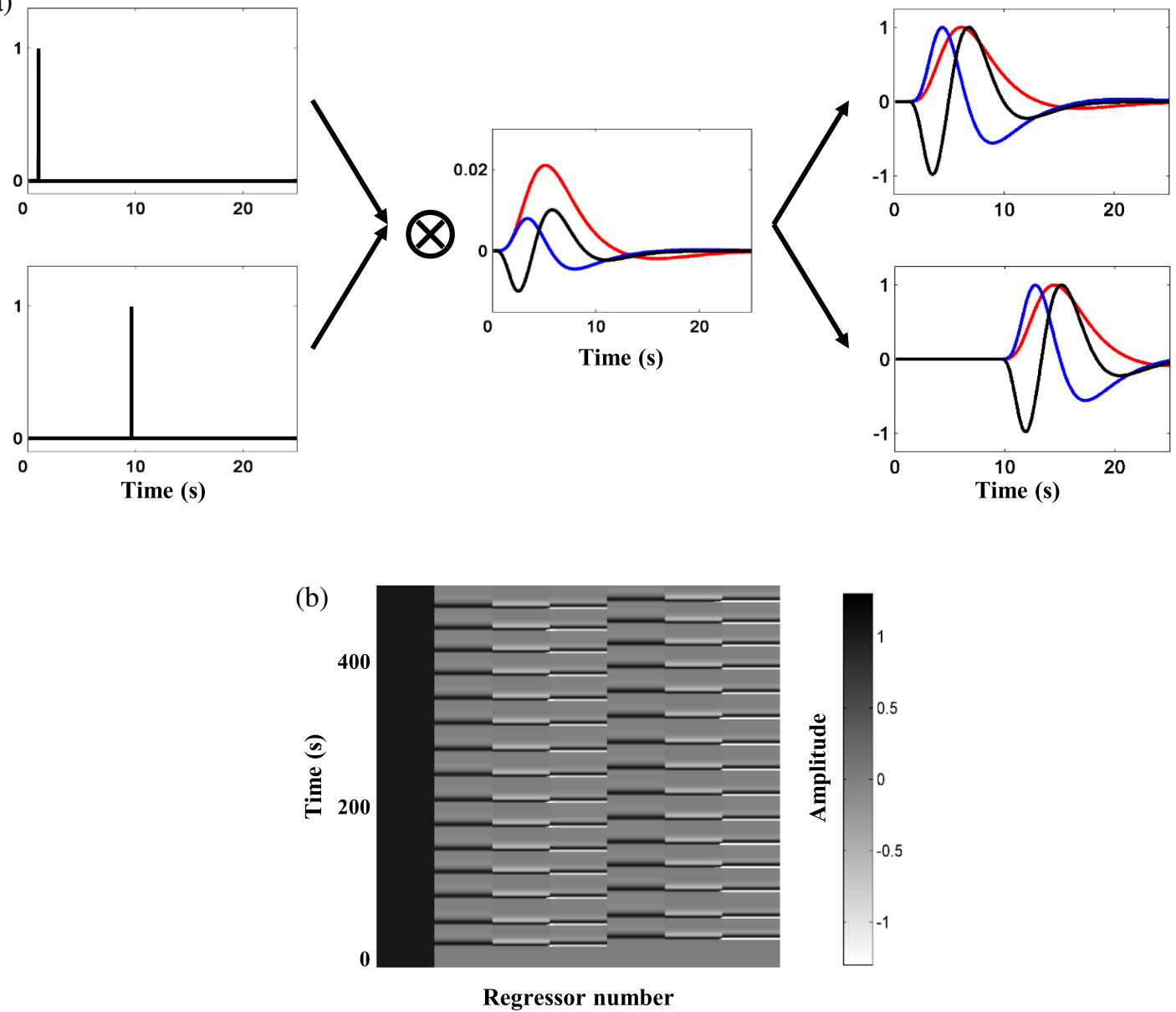

Fig. 4 (a) Construction of neuronal activation regressors based on HRF (red plot for canonical HRF) and its derivatives (temporal and dispersion derivatives; blue and black plots, respectively) convolved with two event-designs of memory encoding and button pressing. (b) An example of regressor matrix design $(x)$ with orders of constant, encoding canonical HRF, encoding temporal, encoding dispersion, buttonpressing canonical, button-pressing temporal, and button-pressing dispersion derivatives.

the strongest activation observed in the right ventrolateral prefrontal cortex (VLPFC, channel 24, right BA 45, $T_{\text {stat }}=9.37$ ). $\mathrm{HHb}$ activation was also investigated in group analysis; however, there was no significant decrease observed in any tasks. Oppositely, we found minor significant $\mathrm{HHb}$ increases in the lower frontopolar only in the spatial WM task. We confirmed the significance of individual data. All subjects (i.e., 16) showed the significantly increased $\mathrm{O}_{2} \mathrm{Hb}$ activation $(p<0.05)$ in right BA 45 (i.e., channels 24). Seven out of 16 subjects showed the significantly decreased $\mathrm{HHb}$ activation $(p<0.05)$ in right BA 45 as performing the spatial WM task. From these results, we classified three response types toward the spatial WM task in BA 45: (1) seven subjects having both significant $\mathrm{O}_{2} \mathrm{Hb}$ increase and $\mathrm{HHb}$ decrease, (2) eight subjects having significant $\mathrm{O}_{2} \mathrm{Hb}$ and $\mathrm{HHb}$ increases, and (3) a subject having significant $\mathrm{O}_{2} \mathrm{Hb}$ increase and null $\mathrm{HHb}$ decrease. This suggested that the $\mathrm{O}_{2} \mathrm{Hb}$ response was more uniform compared to the $\mathrm{HHb}$ response during the spatial WM task. Despite the various hemodynamic responses in the spatial WM task, neither significant $\mathrm{O}_{2} \mathrm{Hb}$ increase nor $\mathrm{HHb}$ decrease was found during control tasks. The finding that the spatial WM task alone showing significant activation suggested that (1) the presence of spontaneous LF systemic noise (and any other noises) does not evoke false-positive results in the group-analysis of control tasks and (2) fNIRS signal during the spatial WM task does reflect encoding-evoked response, neither the sham encoding (i.e., motor control task) nor the motor-related activation (i.e., button-pressing).

\subsection{Temporal Correlation between Prefrontal and Peripheral Signals}

Figure 6 shows the $\mathrm{O}_{2} \mathrm{Hb}$ and $\mathrm{HHb}$ correlations between the fNIRS and shifted peripheral ear signals from a representative subject. The fNIRS signal came from channel 24 located in the right BA 45 in approximate. Because $r_{\max }$ for the $\mathrm{O}_{2} \mathrm{Hb}$ signal [i.e., 0.57; Fig. 6(a)] is greater than the threshold, $\mathrm{O}_{2} \mathrm{Hb}$ denoising was done in that channel. $r_{\max }$ for the $\mathrm{HHb}$ signal is low [i.e., 0.03; Fig. 6(b)]; thus, the $\mathrm{HHb}$ signal of channel 24 was left untouched (red-dotted line). Figure 7 presents the subject-average correlation (inverse $z$-transform) between channelwise fNIRS and peripheral PPG signals $\left(\mathrm{O}_{2} \mathrm{Hb}\right.$ and $\left.\mathrm{HHb}\right)$ for all tasks. There was no significant task effect on both $\left(\mathrm{O}_{2} \mathrm{Hb}\right.$ and $\mathrm{HHb}$ ) prefrontal-periphery correlation and optimum delay $\left(\tau_{\text {opt }}\right)$ using any peripheral sources. The $\mathrm{O}_{2} \mathrm{Hb} \tau_{\text {opt }}$ was more positive (i.e., peripheral signal was ahead of the prefrontal signal, $+0.8 \pm 1.5 \mathrm{~s}$; mean \pm S.D. $)$ than $\mathrm{HHb} \tau_{\mathrm{opt}}(-0.6 \pm 2.2 \mathrm{~s}$; mean \pm S.D.). $\mathrm{O}_{2} \mathrm{Hb}$ signals measured in premotor, temporal, 


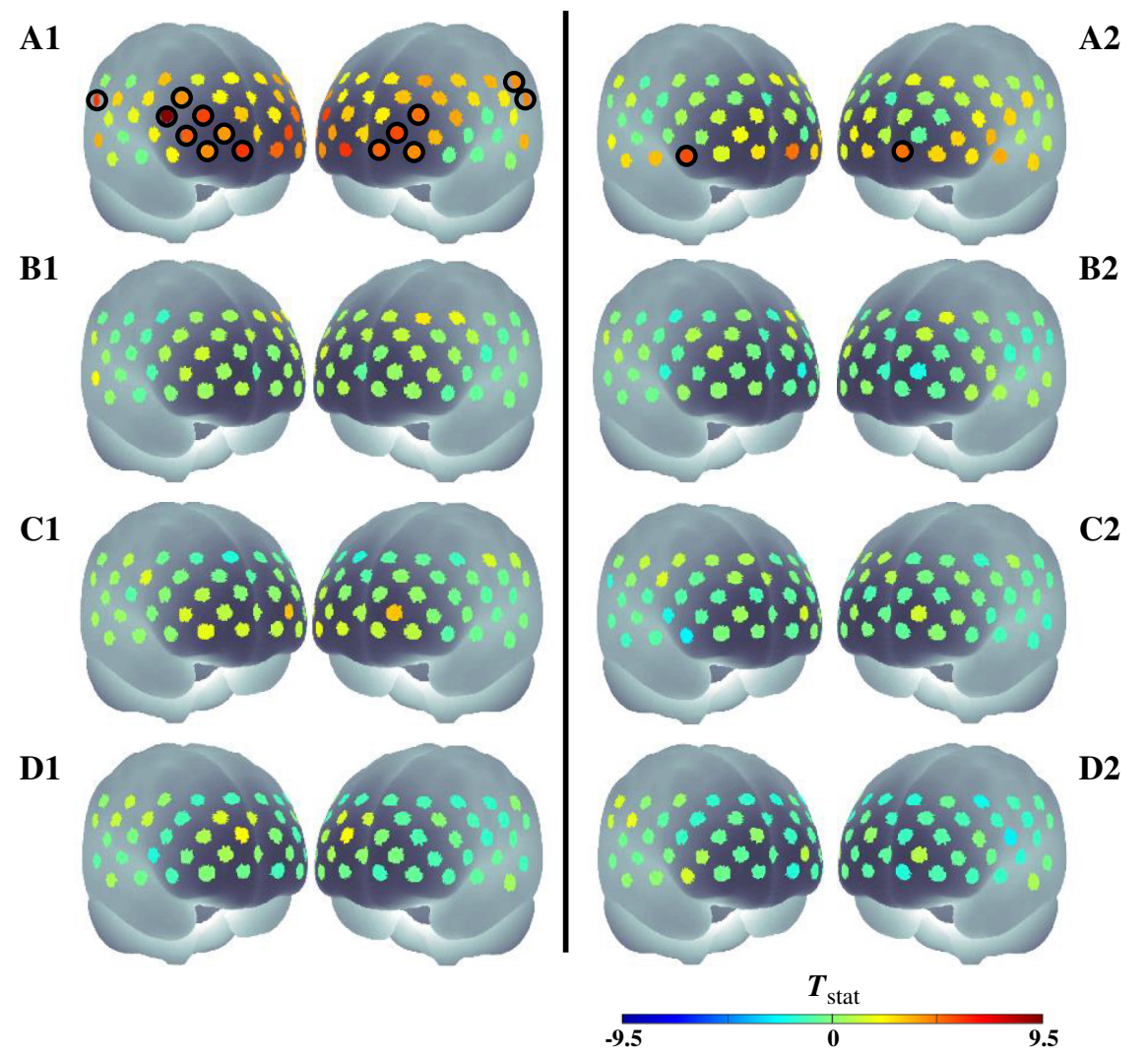

Fig. $5 T_{\text {stat }}$ maps of $\mathrm{O}_{2} \mathrm{Hb}$ (A1-D1) and $\mathrm{HHb}$ (A2-D2) activations for spatial WM (A1-2), motor control (B1-2), eyes-open (C1-2), and eyes-closed RS (D1-2) before denoising. Black encircled channels indicate the channels with significant activation $(p<0.05$; Holm-Bonferroni correction) in one-sample $t$-test.
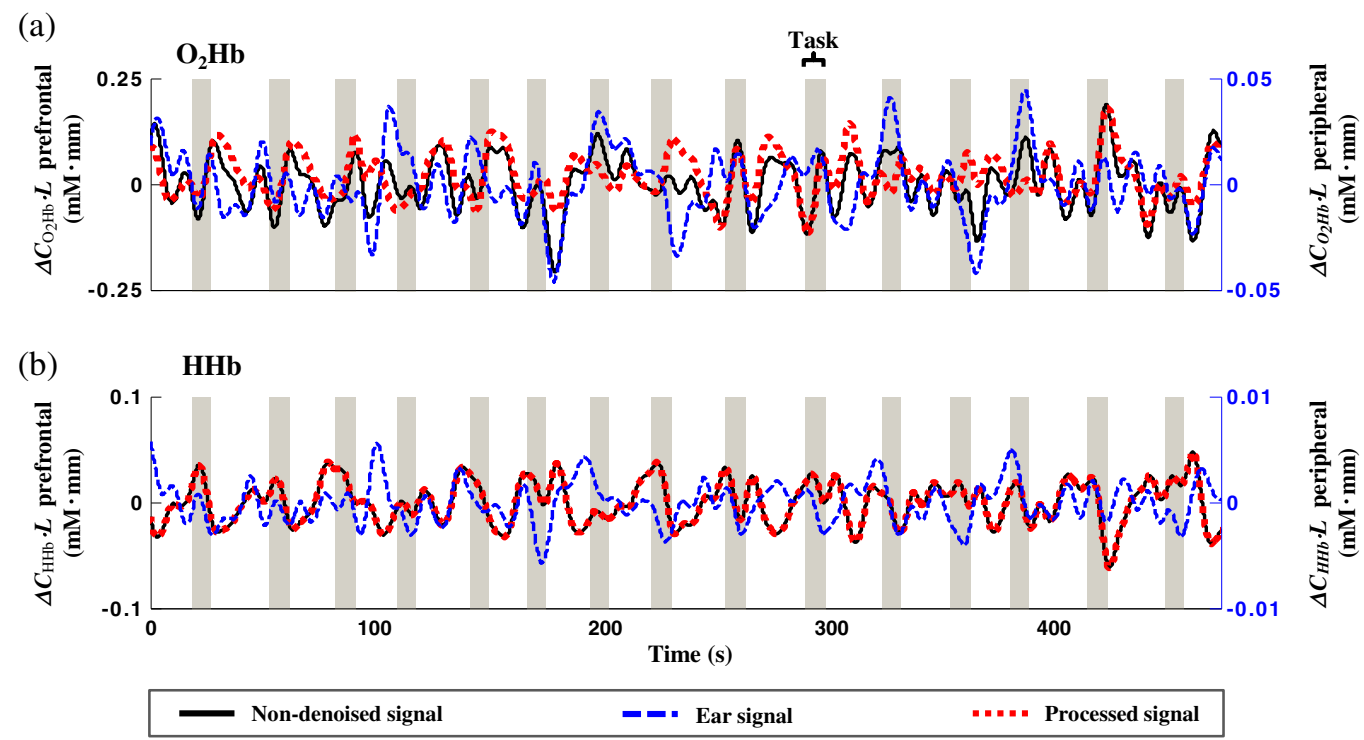

Fig. 6 Representative temporal traces of (a) $\mathrm{O}_{2} \mathrm{Hb}$ and (b) $\mathrm{HHb}$ prefrontal (solid black line, left $y$-axis scale) and peripheral ear (blue dashed-line, right $y$-axis scale) signals illustrating high $\mathrm{O}_{2} \mathrm{Hb}(r=0.57)$ and low $\mathrm{HHb}(r=0.03)$ temporal correlations in the middle frontal cortex of channel 24 during the spatial WM task. Shown peripheral ear signal had already been shifted $\left(+0.7 \mathrm{~s}\right.$ and $-4.3 \mathrm{~s}$ for $\mathrm{O}_{2} \mathrm{Hb}$ and $\mathrm{HHb}$ signals, respectively) according to the corresponding maximum correlation $\left(r_{\max }\right)$. Denoising was performed resulting in the low correlation $(r=-0.13)$ between $\mathrm{O}_{2} \mathrm{Hb}$ denoised (red dotted-line, left $y$-axis scale) and peripheral ear signals. $\mathrm{HHb}$ denoising was not performed due to lower correlation than 0.3 of threshold. Shaded-gray area indicated the task period of spatial WM (target stimulus and delay intervals, $8.5 \mathrm{~s}$ in total). 


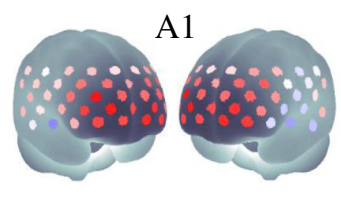

A2
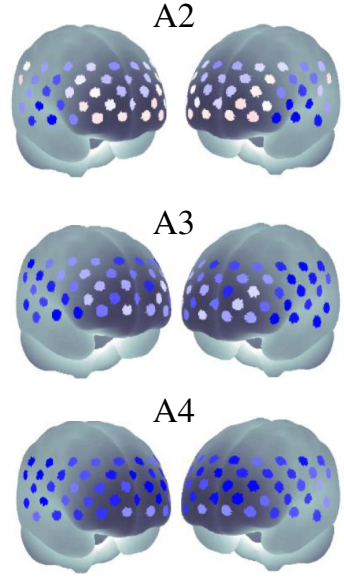
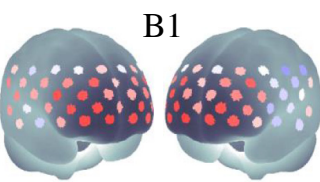

B2
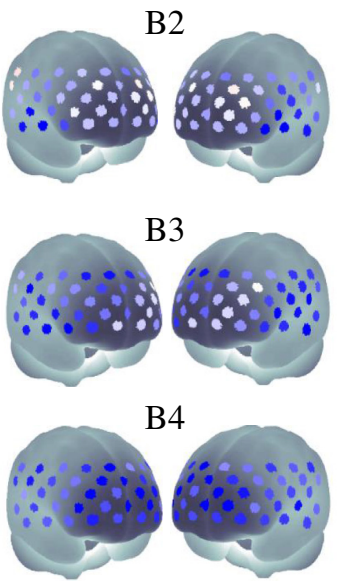
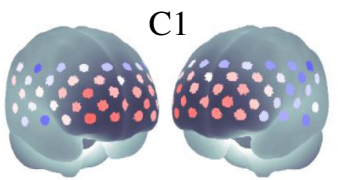

$\mathrm{C} 2$
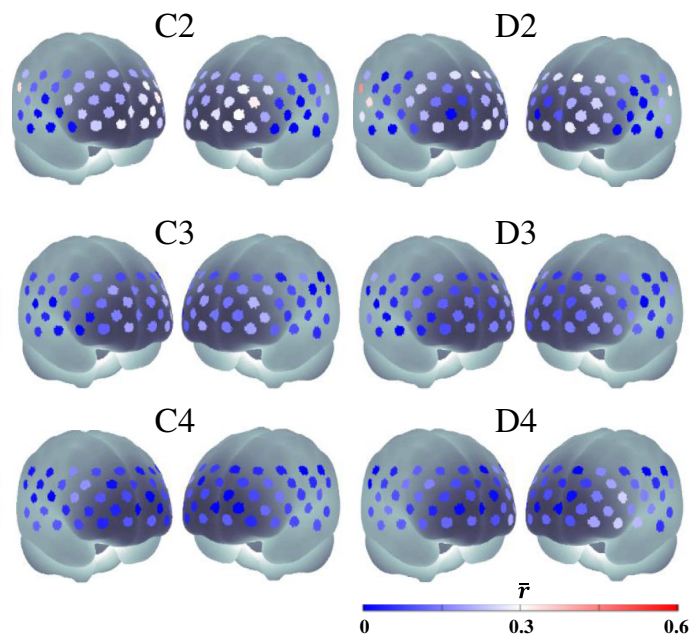

$\mathrm{E}$

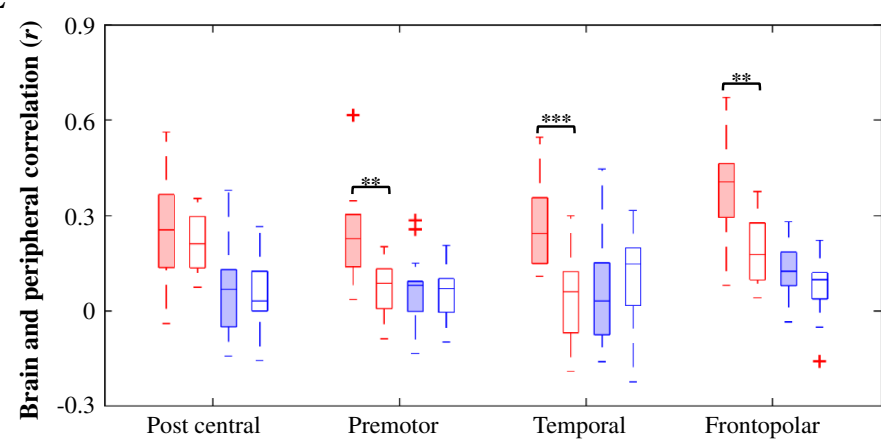

Fig. 7 Subject-average $\mathrm{O}_{2} \mathrm{Hb}(\mathrm{A} 1-\mathrm{D} 2)$ and $\mathrm{HHb}(\mathrm{A} 3-\mathrm{D} 4)$ correlation maps $\left(r_{\text {max }}\right.$; inverse $z$-transform) of prefrontal and peripheral signals measured from ears (A1-D1, A3-D3) and finger (A2-D2, A4-D4) during spatial WM (A1-4), motor control (B1-4), eyes-open (C1-4), and eyes-closed RS (D1-4). $\mathrm{O}_{2} \mathrm{Hb}$ correlation variances across subjects $(E)$ showing significant differences of peripheral sources (red-filled and red-void boxplots for ear and finger signals, respectively) in premotor, temporal, and prefrontal cortices $\left.p<0.001^{(\star \star \star}\right), p<0.01^{(\star \star)}$ for paired-sample $t$-test. There was no observed effect of peripheral sources in $\mathrm{HHb}$ correlations of all regions (blue-filled and blue-void boxplots for ear and finger signals, respectively).

and frontopolar regions had the significantly higher correlation with peripheral signals measured in ears than in fingers [Fig. 7 (e)]. Stronger correlation in the frontopolar was significantly observed compared to premotor (post-hoc Tukey-Kramer test; $p<0.05$ ) for $\mathrm{O}_{2} \mathrm{Hb}$ prefrontal and peripheral ear signals. Meanwhile, peripheral finger signals showed strong relationship with $\mathrm{O}_{2} \mathrm{Hb}$ signals of postcentral and frontopolar regions compared to premotor and temporal regions (post-hoc TukeyKramer test; $p<0.05)$. The selection of peripheral source unlikely influenced $\mathrm{HHb}$ correlations [Figs. 7(A3)-7(D4)] and there was no observed spatial effect (interregions ANOVA, $p>0.05)$. The lower correlation of $\mathrm{HHb}$ prefrontal and peripheral ear signals was significantly observed $(\mathrm{CI}>0.999)$ in all regions compared to the $\mathrm{O}_{2} \mathrm{Hb}$ correlations [i.e., red-filled and blue-filled boxplots in Fig. 7(e)]. $\mathrm{O}_{2} \mathrm{Hb}$ and $\mathrm{HHb}$ correlations between premotor-temporal and peripheral finger signals insignificantly differed. However, postcentral and frontopolar regions showed higher $\mathrm{O}_{2} \mathrm{Hb}$ correlation compared to $\mathrm{HHb}$ correlation toward the peripheral finger signal [i.e., red-void and blue-void boxplots in Fig. 7(e)]. These suggested that (1) HHb signal might be less affected by peripheral shared systemic noise, (2) peripheral ear signals shared more similar components with prefrontal signals than peripheral finger signals, and (3) the effect of systemic noise on $\mathrm{O}_{2} \mathrm{Hb}$ signal was inhomogeneous over the PFC.

\subsection{Efficiency of Denoising}

Denoising performance was compared in all signals (i.e., $\mathrm{O}_{2} \mathrm{Hb}$ and $\mathrm{HHb}$ ) and all tasks (i.e., spatial WM and other control tasks) using the current peripheral-GLM method (i.e., ear, finger, and both signals) and PCA 95. For signal reduction parameter, the task effect was initially evaluated. The signal reduction was comparable across tasks in all signal types and methods. Figure 8 shows subject variances in $\mathrm{O}_{2} \mathrm{Hb}$ and $\mathrm{HHb}$ reductions using several denoising methods corresponding to four major regions. There was no spatial effect (ANOVA, $p>0.05$ ) on $\mathrm{O}_{2} \mathrm{Hb}$ and $\mathrm{HHb}$ reductions using PCA 95. Performance of $\mathrm{O}_{2} \mathrm{Hb}$ reduction using the ear-GLM denoising was spatially related where the highest reduction happened in the frontopolar ( $34 \pm 16 \%$, task-average max. in left BA $10 / 11$ by $45 \pm 23 \%$; mean \pm S.D.). Peripheral-GLM denoising using ear signals had 
(a)

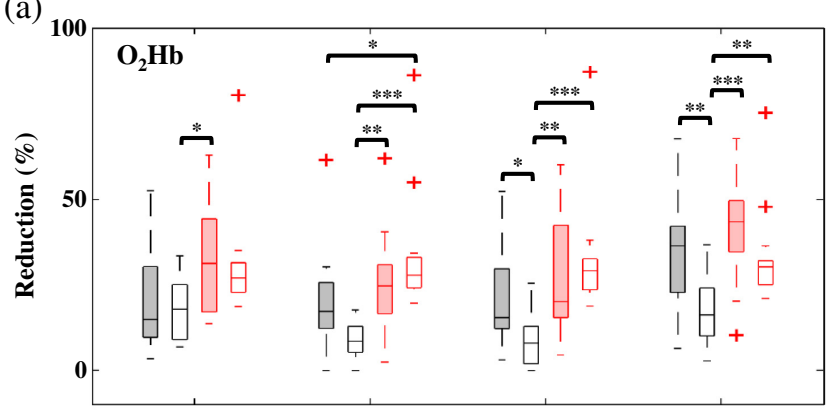

(b)

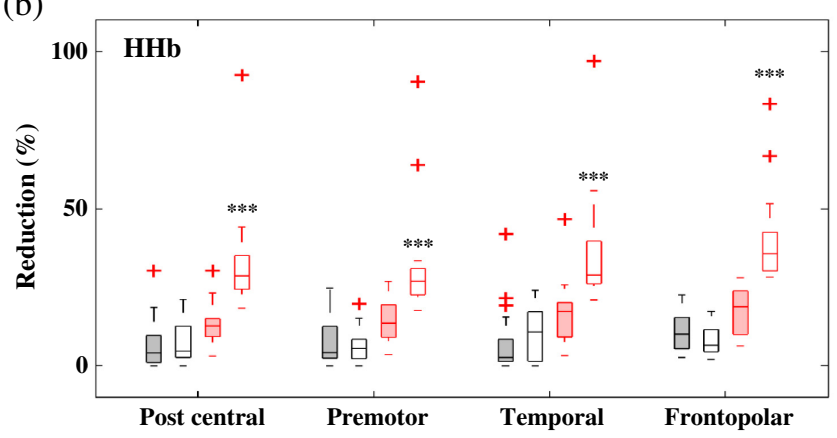

Fig. 8 Subject variances of systemic noise reduction in (a) $\mathrm{O}_{2} \mathrm{Hb}$ and (b) $\mathrm{HHb}$ signals neglecting the task effect in four channel regions: postcentral, premotor, temporal, and prefrontal cortices performed by ear-GLM denoising (black-filled boxplot), finger-GLM denoising (black-void boxplot), dual peripheral-GLM denoising (red-filled boxplot), and PCA 95 (red-void boxplot) $-p<0.001^{(* *)}, p<0.01^{(* *)}$, $p<0.05^{(*)}$ for ANOVA and post-hoc Tukey-Kramer test. Because of low correlations between prefrontal and peripheral signals in $\mathrm{HHb}$ parameter, $\mathrm{HHb}$ signals were mostly not denoised. Therefore, the performance of peripheral-GLM denoising in $\mathrm{HHb}$ signal was significantly lower than PCA 95.

comparable $\mathrm{O}_{2} \mathrm{Hb}$ noise reduction to PCA 95 in all regions except premotor (post-hoc Tukey-Kramer test). By using both ear and finger signals, the denoising performance insignificantly differed compared to PCA 95. The performance of peripheral finger regressor was worse compared to the dual ear-finger regressors in all regions. $\mathrm{HHb}$ prefrontal and any peripheral signals presented low correlation in all regions (i.e., no spatial effect); thus, $\mathrm{HHb}$ signals were likely left unprocessed. Therefore, denoising performance of $\mathrm{HHb}$ peripheral-GLM method was significantly lower than PCA 95.

We consistently found no activation and no substantial effect size change of $\mathrm{O}_{2} \mathrm{Hb}$ and $\mathrm{HHb}$ activations in control tasks after denoising using any methods. The denoising effect on activation changes was only observed in the spatial WM task. Figure 9 shows Cohen's $d$ effect size maps of $\mathrm{O}_{2} \mathrm{Hb}$ and $\mathrm{HHb}$ activations during the spatial WM task after denoising. Denoising decreased the significances of $\mathrm{O}_{2} \mathrm{Hb}$ increase ( $p<0.05$; HolmBonferroni correction; black-circled channels) in group-analysis. The most decrease was performed by the dual-peripheralGLM denoising [Fig. 9(D1)] showing more right lateralization (i.e., BA 45; channel 24). By using only ear signals as noise regressors, we still observed $\mathrm{O}_{2} \mathrm{Hb}$ significance in the surrounding BA 45 compared to the finger-GLM and dual peripheralGLM denoising. Regarding the low reduction of $\mathrm{O}_{2} \mathrm{Hb}$ signal by finger-GLM denoising [Fig. 8(a)], the decreased significance of $\mathrm{O}_{2} \mathrm{Hb}$ activation was unexpectedly observed. Furthermore, the change of $\mathrm{HHb}$ activation was not observed after denoising using any methods [Figs. 9(A2)-9(E2)]. These results were expected in the peripheral-GLM denoising due to low signal reduction; yet, PCA 95 presenting comparable signal reduction in both $\mathrm{O}_{2} \mathrm{Hb}$ and $\mathrm{HHb}$ signals also showed the insignificant change of $\mathrm{HHb}$ activation. There was no significant difference of channelwise activation (i.e., $\mathrm{O}_{2} \mathrm{Hb}$ and $\mathrm{HHb}$ ) across methods.

\section{Discussion}

\subsection{Direct Versus Multimodality Interpretations}

Some physiological measurements, such as respiration rate by a pneumatic belt or arterial blood pressure by cuff, ${ }^{45,65}$ require additional interpretations to translate them into blood-related LF signals, before used as noise regressors in fNIRS studies. In our study, we directly measured the blood-related LF signals in peripheral sites using PPG, concurrently with the prefrontal fNIRS measurement. The peripheral LF signal is so-called "catch-all" signal that represents the combination of LF systemic physiological noises from various origins. It may include the fluctuations in heart rate (HR), arterial blood pressure (ABP), respiration (or downsampled version of them). Under the assumption that these signals affect both brain and peripherals based on vascular relations, the blood-related physiological signals can be used directly to denoise functional signals (i.e., fNIRS or fMRI) without knowing exactly the origins of these low-frequency oscillations (LFOs). The detailed benefits of such direct measurement can be found in the previous work of Tong et al. through simultaneous fMRI-NIRS measurement. ${ }^{49,66}$

\subsection{Regressor Suitability}

Cooper et al. ${ }^{67}$ previously used frontal and temporal lobes fNIRS signals as physiological regressors for fMRI data. However, an fNIRS signal with $3 \mathrm{~cm}$ of S-D distance is a mixture of both neuronal and nonneuronal signals from cortical and extracerebral layers, respectively. Directly using those as models could regress out the real neuronal signal. Concurrent finger measurements have been attempted to obtain the neuronalfree (i.e., systemic) signal as a noise regressor. ${ }^{49,66}$ However, noise regressor obtained from the ear has the following advantages: (1) the blood in ear is supplied by external carotid artery, which is close to the internal carotid artery (i.e., blood supply to brain); (2) the ear is easy to measure by our clip-equipped PPG probe with superior signal-to-noise ratio. As shown in Fig. 7, peripheral ear signals had the significantly higher correlation with prefrontal signals than those from fingers resulting in greater noise reduction (Fig. 8). This confirms our hypothesis that closer vascular distance may lead to high similarities between the signals. Despite the less signal reduction, the significance of $\mathrm{O}_{2} \mathrm{Hb}$ activation in group analysis decreased after finger-GLM denoising. This suggested that denoising using less correlated signals could possibly regress out some signal portions. It was difficult to evaluate whether over-processing (i.e., spurious improved activation localization) actually happened or not. Therefore, the highly correlated noise model (e.g., peripheral ear signals) is more preferable for denoising.

In addition to sources of systemic noise regressor, we also investigated the regressor suitability between two signal types $\left(\mathrm{O}_{2} \mathrm{Hb}\right.$ versus $\left.\mathrm{HHb}\right)$. The correlation between $\mathrm{HHb}$ prefrontal and peripheral (either ear or finger) signals is significantly lower than that of $\mathrm{O}_{2} \mathrm{Hb}$ signals (Fig. 7). There are two arguments that may explain this $\mathrm{HHb}$ characteristic. First, $\mathrm{HHb}$ is less affected by the systemic noise. For example, according 
A1

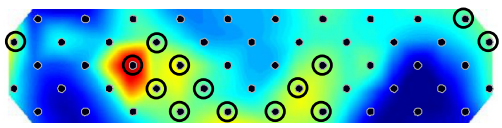

B1

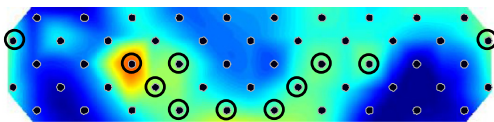

C1

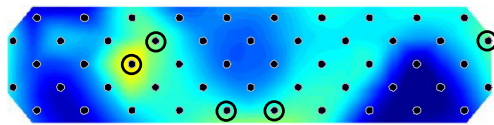

D1

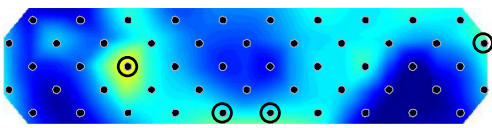

E1

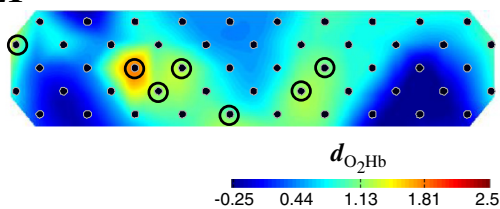

A2

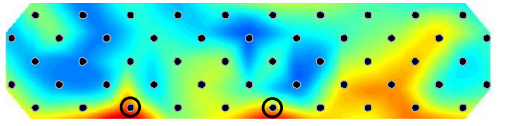

B2

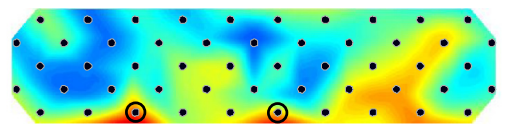

C2

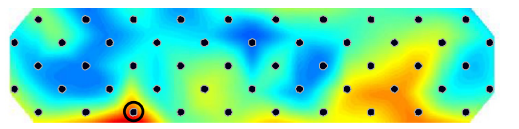

D2

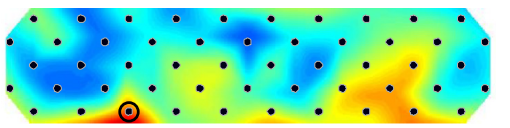

E2

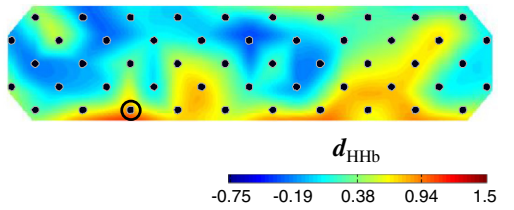

Fig. 9 Cohen's $d$ effect size maps of $\mathrm{O}_{2} \mathrm{Hb}$ (A1-E1) and $\mathrm{HHb}$ (A2-E2) activations for the spatial WM task before denoising (A1-2), after ear-GLM denoising (B1-2), finger-GLM denoising (C1-2), dual peripheralGLM denoising (D1-2), and PCA 95 (E1-2). Black encircled channels indicate the significant activation channels $(p<0.05$; Holm-Bonferroni correction).

to Franceschini et al., ${ }^{18}$ heart rate change significantly modulates the arterial compartment and is highly reflected in $\mathrm{O}_{2} \mathrm{Hb}$; this is not the case for $\mathrm{HHb}$ in venous vasculature. Therefore, the $\mathrm{HHb}$ signal may be less prone to those systemic physiological fluctuations. This hypothesis is also consistent with the results reported by Sato et al. ${ }^{68}$ with the finding of lower correlation between $\mathrm{HHb}$ signal at brain and systemic skin blood flow (SBF) measured using laser Doppler flowmeter. Second, neither peripheral nor systemic SBF recordings are sufficient to model systemic noise in $\mathrm{HHb}$ signal. Even $\mathrm{HHb}$ extracerebral was found having low coherence with $\mathrm{HHb}$ brain. ${ }^{69}$ Previous studies reported that $\mathrm{HHb}$ brain is less sensitive and inconsistently responds toward cerebral blood flow change. ${ }^{70,71}$ In the current results, we found nonuniform $\mathrm{HHb}$ responses during the encoding interval across subjects resulting in the insignificance of $\mathrm{HHb}$ activation. The individual variances may implicate this issue. According to the above arguments, a global systemic variation in $\mathrm{O}_{2} \mathrm{Hb}$ signal is a better noise regressor.

\subsection{Systemic Denoising and Its Comparison to Other Denoising Methods}

Katura et al. ${ }^{72}$ demonstrated the causal relationship between fNIRS signals and cardiovascular systemic noises modeled using HR and mean ABP during resting state. The study focused on a single channel signal around the posterior-superior temporal lobe and reported that the contribution of cardiovascular systemic noise up to $35 \%$ in $\mathrm{O}_{2} \mathrm{Hb}$ and $\mathrm{Hb}$-total signals. The remaining $65 \%$ portion was still unidentified noise components. In this study, we sought to isolate and remove systemic noise identified from peripheral sites. The reduction caused by systemic denoising (i.e., peripheral ear in $\mathrm{O}_{2} \mathrm{Hb}$ signal), or also called as systemic noise contribution, was found to be around $25 \%$ to $34 \%$ in average and $39 \%$ to $58 \%$ in maximum (frontopolar channel 36; BA 10/11) across tasks.

From our results, we found that the systemic noise contribution was equal for any tasks. The spatial variability of systemic noise contribution was also observed over PFC as previously reported. ${ }^{73}$ Zhang et al. ${ }^{74}$ showed that LFO highly accumulated in $\mathrm{O}_{2} \mathrm{Hb}$ signal over the frontopolar regions. This phenomenon may be explained by the fact that low signal sensitivity in the regions with big vessels (causing high absorption and low photon escape $\mathrm{e}^{75,76}$ ). While the frontopolar is covered by the relatively small vessels ( 0.9 to $1.1 \mathrm{~mm}$ in diameter), ${ }^{77}$ the vessels in temporal regions are slightly greater than $2 \mathrm{~mm}^{78}$ Apart from the above arguments, the task-evoked systemic noise might be colocalized in the draining scalp veins. ${ }^{46,49}$

In order to quantify the comparison between current and previous methods, we performed PCA 95 in the same datasets. PCA 95 is a well-known method to eliminate motion artifact with the assumption of less contribution of noise toward the signal. The arbitrary threshold of contribution cut-off (e.g., 95\% and $80 \%$ ) always become a limitation of PCA. Performance of PCA in signal reduction was uniform over PFC while the performance of peripheral-GLM denoising was spatially influenced. The $\mathrm{O}_{2} \mathrm{Hb}$ signal reduction was comparable between (dual) peripheral-GLM denoising and PCA. After denoising, the significance of $\mathrm{O}_{2} \mathrm{Hb}$ activation in group analysis was decreased by all the methods. There was no channelwise difference across methods in both $\mathrm{O}_{2} \mathrm{Hb}$ and $\mathrm{HHb}$ activations despite the high reduction of $\mathrm{HHb}$ signal by PCA 95. This could suggest that (1) the current peripheral-GLM denoising performed 
comparably to PCA 95 in $\mathrm{O}_{2} \mathrm{Hb}$ signals and (2) the HRF model for $\mathrm{HHb}$ signals might not be optimum. Uga et al. ${ }^{79}$ reported that the optimization of $\tau_{p}$ parameter could improve the observations of $\mathrm{O}_{2} \mathrm{Hb}$ increase and $\mathrm{HHb}$ decrease. Even though the performance of peripheral-GLM denoising was equal to PCA, our current method could specifically remove systemic (i.e., nonneuronal) components rather than determining the assumption of signal contribution as PCA. Furthermore, PCA performance is influenced by the availability of channel number. Peripheral GLM denoising is potentially applicable for the required minimum measurement system.

Despite the potential results, we were still aware of the extracerebral (e.g., scalp) signal in the denoised signals. The scalp effect has been reported to be significant in PFC. ${ }^{80}$ One of the well-known methods to eliminate extracerebral signal is MD S-D separation. ${ }^{36}$ The characteristics of systemic denoising and MD S-D separation can be different from each other. External carotid artery supplying blood to ear shares the same upstream vasculature (i.e., common carotid artery) with internal carotid artery for brain supply. Any fluctuations in common carotid artery could affect downstream vessels in both brain and ears. Thus, the ear measurement is a reasonable interpretation of global systemic noise. In opposite, the MD S-D separation directly measures the regional changes in the superficial layer corresponding to nearby fNIRS channels. We believe that global systemic noise is also confined in the regional superficial signal due to similar blood vasculature as mentioned above. The impact of global systemic noise on regional superficial signal is still unknown. Furthermore, both systemic and extracerebral denoising have not yet been done together in
fNIRS data analysis. We hope to address this issue in the future studies.

\subsection{Task-Related Effect on Peripheral Ear Signals}

There are two issues concerned with this subdiscussion. First, we have observed the high correlation between the $\mathrm{O}_{2} \mathrm{Hb}$ frontopolar and peripheral ear signals for all tasks. The correlation distributions were also similar regardless of tasks (Fig. 7). Tachtsidis et al. and Sato et al. ${ }^{68,81}$ evidenced the high correlation of frontal lobe $\mathrm{O}_{2} \mathrm{Hb}$ fNIRS with global changes of mean blood pressure and SBF, respectively. Kohno et al. ${ }^{40}$ also reported the effect of SBF contamination on the wide area of the forehead. This may implicate the high correlation between $\mathrm{O}_{2} \mathrm{Hb}$ peripheral ear and SBF. This relationship is likely associated with the same vascular source of the common carotid artery.

Second, we explored the task-related signals from peripheral measurements as shown in Figs. 10(A1)-10(D2). The averaged waveforms were different for all tasks. As performing the same task, ear and finger signals presented difference waveforms. $\mathrm{Li}$ et al. ${ }^{82}$ demonstrated the sensitivity difference on systemic manipulation responses (e.g., passive leg raising, paced breathing) in the ear, finger, and toe. We confirmed that there was no significant difference in $\mathrm{O}_{2} \mathrm{Hb}$ and $\mathrm{HHb}$ activations between eyes-open and eyes-closed RS controls in both peripheral ear and finger signals. The significant task-related effect was observed in both prefrontal and peripheral (i.e., ear and finger) signals during the spatial WM task [Figs. 10(A1)-10(A3)]. Such effect was also reported in other tasks: verbal WM, finger
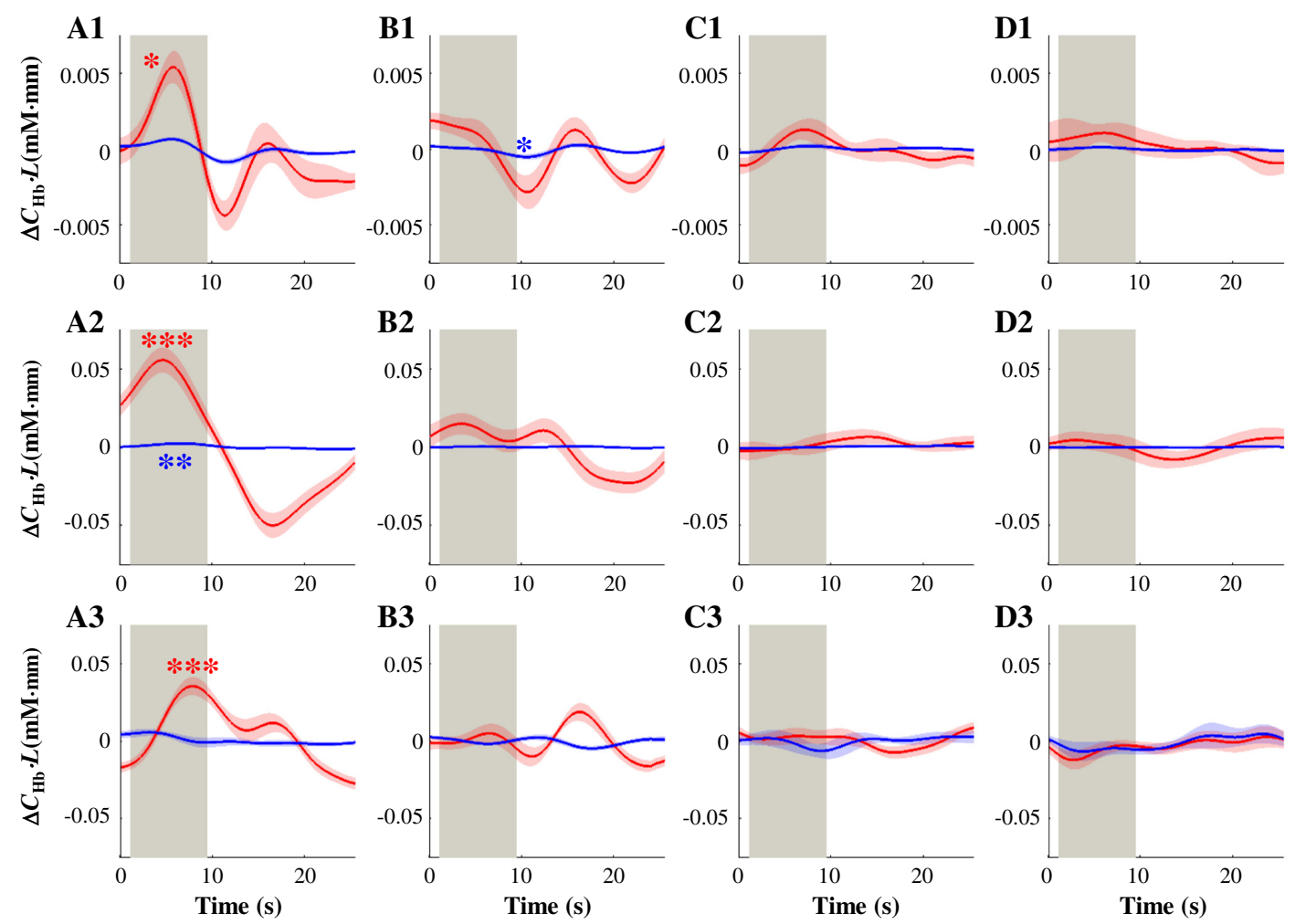

Fig. 10 Subject-average ear (A1-D1), finger (A2-D2), and right BA 45 (channel 24, A3-D3) signals (red and blue lines for $\mathrm{O}_{2} \mathrm{Hb}$ and $\mathrm{HHb}$ signals, respectively, with their standard errors) during (a) spatial WM, (b) motor control, (c) eyes-open, and (d) eyes-closed RS. The gray-shaded interval indicates the task period (target stimulus and delay intervals, $8.5 \mathrm{~s}$ in total). $P<0.01^{(* *)}, p<0.05^{(* *)}$, and $p<0.1^{(*)}$ for one-sample $t$-test of $\mathrm{O}_{2} \mathrm{Hb}$ increase and $\mathrm{HHb}$ decrease activations ( $\beta$ of encoding canonical HRF). 
tapping, visual checker-board, and anagram tasks. ${ }^{36,68,81}$ This finding is also consistent with the previous work of Kirilina et al., ${ }^{46}$ where they found task-related activations in the superficial layer and concluded that "physiological origin of the systemic artifact is a task-evoked sympathetic arterial vasoconstriction followed by a decrease in venous volume." These results comprehensively suggested that the tasks also simultaneously triggered the global systemic noises, which is consistent with the neurovascular coupling theorem arguing the local systemic regulation. ${ }^{4,83,84}$

Since the peripheral signals have task-evoked effects, there is a concern about the denoising method using the peripheral signals. We argue that the signal from the ears is a pure systemic physiological artifact. It does not contain any neuronal "contamination," even it shows task-evoked changes. These changes are likely induced by global systemic changes as a result of performing tasks. Therefore, these peripheral signals are "noises" and have to be removed, regardless of their waveforms. Moreover, their waveforms (i.e., global systemic changes) should be different from the real brain activations, which is neuronal and regional [Figs. 10(A3)-10(D3)]. These global systemic changes are likely coupled and propagated into the blood-related change (i.e., flow, volume, and oxygenation) that affects the different brain and facial regions at the different time. Thus, in this study, in addition to accurately record the non-neuronal noise from ears, we adaptively remove it by temporally matching with each fNIRS channel before subtraction. As a result, we found the significant $\mathrm{O}_{2} \mathrm{Hb}$ increase in DLPFC/ VLPFC during the spatial WM task (Fig. 9) as previous studies ${ }^{85-88}$ even after denoising. This demonstrates that removing physiological noise with task-related waveform, we did not remove the task activation.

\section{Conclusion}

A high correlation between $\mathrm{O}_{2} \mathrm{Hb}$ prefrontal and peripheral signals was observed consistently during all tasks, especially the peripheral signals from ears. Even without denoising process, $\mathrm{O}_{2} \mathrm{Hb}$ activation was only detected in the spatial WM task. This suggested that the risks of false-positive activation in control tasks were rejected and fNIRS did measure the task-evoked activation. Although the task-evoked effect also globally appeared in the peripheral signals, the real neuronal task activation still remained after denoising. This denoising method had reduced the systemic noise up to $39 \%$ to $58 \%$ of raw signals. The denoising performance was comparable to PCA 95 and offered the advantages of specific systemic (i.e., nonneuronal) noise model, minimum measurement system (i.e., a peripheral channel versus multiprefrontal channels), and no required assumption of component contribution. Despite these promising results, the extracerebral noise that may regulate locally was not considered. Both global systemic and regional extracerebral noises should be managed to improve the analysis accuracy and this issue will be addressed in the future studies.

\section{Disclosures}

The authors have no relevant financial interests in this article and no potential conflicts of interest to disclose.

\section{Acknowledgments}

The work was supported by the National Institutes of Health under Grant Nos. K25 DA031769 (Y.T.) and R21 DA032746, R01 NS097512 (B.deB.F). T.B. Tang was funded by Ministry of
Education under HICoE grant scheme to Centre for Intelligent Signal and Imaging Research, Universiti Teknologi PETRONAS.

\section{References}

1. A. Villringer et al., "Near infrared spectroscopy (NIRS): a new tool to study hemodynamic changes during activation of brain function in human adults," Neurosci. Lett. 154(1-2), 101-104 (1993).

2. A. Maki et al., "Spatial and temporal analysis of human motor activity using noninvasive NIR topography," Med. Phys. 22(12), 1997-2005 (1995).

3. F. Scholkmann et al., "A review on continuous wave functional nearinfrared spectroscopy and imaging instrumentation and methodology," NeuroImage 85(1), 6-27 (2014).

4. D. Malonek et al., "Vascular imprints of neuronal activity: relationships between the dynamics of cortical blood flow, oxygenation, and volume changes following sensory stimulation," Proc. Natl. Acad. Sci. U. S. A. 94(26), 14826-14831 (1997).

5. A. Moreno et al., "Neurophysiological, metabolic and cellular compartments that drive neurovascular coupling and neuroimaging signals," Front. Neuroenerg. 5, 3 (2013).

6. M. Ferrari and V. Quaresima, "A brief review on the history of human functional near-infrared spectroscopy (fNIRS) development and fields of application," NeuroImage 63(2), 921-935 (2012).

7. A. Villringer and B. Chance, "Non-invasive optical spectroscopy and imaging of human brain function," Trends Neurosci. 20(10), 435-442 (1997).

8. H. Koizumi et al., "Optical topography: practical problems and new applications," Appl. Opt. 42(16), 3054-3062 (2003).

9. R. N. Aslin and J. Mehler, "Near-infrared spectroscopy for functional studies of brain activity in human infants: promise, prospects, and challenges," J. Biomed. Opt. 10(1), 011009 (2005).

10. H. Sato et al., "Cerebral hemodynamics in newborn infants exposed to speech sounds: a whole-head optical topography study," Hum. Brain Mapp. 33(9), 2092-2103 (2012).

11. Y. Monden et al., "Individual classification of ADHD children by right prefrontal hemodynamic responses during a go/no-go task as assessed by fNIRS," NeuroImage 9, 1-12 (2015).

12. K. Yamamuro et al., "Prefrontal dysfunction in pediatric Tourette's disorder as measured by near-infrared spectroscopy," BMC Psychiatry 15, 102 (2015).

13. M. Suzuki et al., "Prefrontal and premotor cortices are involved in adapting walking and running speed on the treadmill: an optical imaging study," NeuroImage 23(3), 1020-1026 (2004).

14. H. Atsumori et al., "Noninvasive imaging of prefrontal activation during attention-demanding tasks performed while walking using a wearable optical topography system," J. Biomed. Opt. 15(4), 046002 (2010).

15. L. Pollonini et al., "Auditory cortex activation to natural speech and simulated cochlear implant speech measured with functional nearinfrared spectroscopy," Hear. Res. 309, 84-93 (2014).

16. C. Mckay et al., "Connectivity in language areas of the brain in the cochlear implant users as revealed by fNIRS," Adv. Exp. Med. Biol. 894, 327-335 (2016).

17. V. Toronov et al., "Near-infrared study of fluctuations in cerebral hemodynamics during rest and motor stimulation: temporal analysis and spatial mapping," Med. Phys. 27(4), 801-815 (2000).

18. M. A. Franceschini et al., "Hemodynamic evoked response of the sensorimotor cortex measured noninvasively with near-infrared optical imaging," Psychophysiology 40(4), 548-560 (2003).

19. H. Obrig et al., "Spontaneous low frequency oscillations of cerebral hemodynamics and metabolism in human adults," NeuroImage 12(6), 623-639 (2000).

20. Y. Tong and B. D. Frederick, "Time lag dependent multimodal processing of concurrent fMRI and near-infrared spectroscopy (NIRS) data suggests a global circulatory origin for low-frequency oscillation signals in human brain," NeuroImage 53(2), 553-564 (2010).

21. J. E. W. Mayhew et al., "Cerebral vasomotion: a $0.1-\mathrm{Hz}$ oscillation in reflected light imaging of neural activity," NeuroImage 4(3), 183-193 (1996).

22. R. Zhang, J. H. Zuckerman, and B. D. Levine, "Spontaneous fluctuations in cerebral blood flow: insights from extended-duration recordings 
in humans," Am. J. Physiol. Heart Circ. Physiol. 278(6), H1848-H1855 (2000).

23. D. A. Boas, A. M. Dale, and M. A. Franceschini, "Diffuse optical imaging of brain activation: approaches to optimizing image sensitivity, resolution, and accuracy," Neurolmage 23(Suppl. 1), S275-S288 (2004).

24. S. Coyle, T. Ward, and C. Markham, "Physiological noise in near-infrared spectroscopy: implications for optical brain computer interfacing," in 26th Annual Int. Conf. IEEE Engineering Medicine and Biology Society, Vol. 6, pp. 4540-4543 (2004).

25. E. Kirilina et al., "Identifying and quantifying main components of physiological noise in functional near infrared spectroscopy on the prefrontal cortex," Front. Hum. Neurosci. 7, 864 (2013).

26. J. Tanji and E. Hoshi, "Role of the lateral prefrontal cortex in executive behavioral control," Physiol. Rev. 88(1), 37-57 (2008).

27. Y. Moriguchi and K. Hiraki, "Prefrontal cortex and executive function in young children: a review of NIRS studies," Front. Hum. Neurosci. 7, 1-9 (2013).

28. A. Sassaroli et al., "Data analysis and statistical tests for near-infrared functional studies of the brain," Proc. SPIE 6850, 685008 (2008).

29. A. Eklund, T. E. Nichols, and H. Knutsson, "Cluster failure: why fMRI inferences for spatial extent have inflated false-positive rates," Proc. Natl. Acad. Sci. U. S. A. 113(28), 7900-7905 (2016).

30. L. M. Hocke et al., "Comparison of peripheral near-infrared spectroscopy low-frequency oscillations to other denoising methods in resting state functional MRI with ultrahigh temporal resolution," Magn. Reson. Med. 76(6), 1697-1707 (2016).

31. R. B. Saager and A. J. Berger, "Direct characterization and removal of interfering absorption trends in two-layer turbid media," J. Opt. Soc. Am. A 22(9), 1874-1882 (2005).

32. R. Saager and A. Berger, "Measurement of layer-like hemodynamic trends in scalp and cortex: implications for physiological baseline suppression in functional near-infrared spectroscopy," J. Biomed. Opt. 13(3), 034017 (2008).

33. L. Gagnon et al., "Double-layer estimation of intra- and extracerebral hemoglobin concentration with a time-resolved system," J. Biomed. Opt. 13(5), 054019 (2008).

34. R. B. Saager, N. L. Telleri, and A. J. Berger, "Two-detector corrected near infrared spectroscopy (C-NIRS) detects hemodynamic activation responses more robustly than single-detector NIRS," NeuroImage 55(4), 1679-1685 (2011).

35. S. B. Erdoğan, M. A. Yücel, and A. Akin, "Analysis of task-evoked systemic interference in fNIRS measurements: insights from fMRI," NeuroImage 87, 490-504 (2014).

36. T. Funane et al., "Quantitative evaluation of deep and shallow tissue layers' contribution to fNIRS signal using multi-distance optodes and independent component analysis," NeuroImage 85(1), 150-165 (2014).

37. T. Yamada, S. Umeyama, and K. Matsuda, "Separation of fNIRS signals into functional and systemic components based on differences in hemodynamic modalities," PLoS One 7(11), e50271 (2012).

38. S. Prince et al., "Time-series estimation of biological factors in optical diffusion tomography," Phys. Med. Biol. 48(11), 1491-1504 (2003).

39. Y. Zhang et al., "Eigenvector-based spatial filtering for reduction of physiological interference in diffuse optical imaging," J. Biomed. Opt. 10(1), 011014 (2005).

40. S. Kohno et al., "Removal of the skin blood flow artifact in functional near-infrared spectroscopic imaging data through independent component analysis," J. Biomed. Opt. 12(6), 062111 (2007).

41. T. Katura et al., "Extracting task-related activation components from optical topography measurement using independent components analysis," J. Biomed. Opt. 13(5), 054008 (2008).

42. J. Markham et al., "Blind identification of evoked human brain activity with independent component analysis of optical data," Hum. Brain Mapp. 30(8), 2382-2392 (2009).

43. J. Virtanen, T. Noponen, and P. Merilainen, "Comparison of principal and independent component analysis in removing extracerebral interference from near-infrared spectroscopy signals," J. Biomed. Opt. 14(5), 054032 (2009).

44. H. Tanaka, T. Katura, and H. Sato, "Task-related oxygenation and cerebral blood volume changes estimated from NIRS signals in motor and cognitive tasks," Neurolmage 94(1), 107-119 (2014).
45. R. M. Birn et al., "Separating respiratory-variation-related fluctuations from neuronal-activity-related fluctuations in fMRI," NeuroImage 31(4), 1536-1548 (2006).

46. E. Kirilina et al., "The physiological origin of task-evoked systemic artefacts in functional near infrared spectroscopy," NeuroImage 61(1), 70-81 (2012).

47. J. C. Ye et al., "NIRS-SPM: statistical parametric mapping for nearinfrared spectroscopy," NeuroImage 44(2), 428-447 (2009).

48. S. Tak and J. C. Ye, "Statistical analysis of fNIRS data: a comprehensive review," NeuroImage 85, 72-91 (2014).

49. Y. Tong et al., "Low-frequency oscillations measured in the periphery with near-infrared spectroscopy are strongly correlated with blood oxygen level-dependent functional magnetic resonance imaging signals," J. Biomed. Opt. 17(10), 106004 (2012).

50. R. C. Oldfield, "The assessment and analysis of handedness: the Edinburgh inventory," Neuropsychologia 9(1), 97-113 (1971).

51. R. Aoki et al., "Relationship of negative mood with prefrontal cortex activity during working memory tasks: an optical topography study," Neurosci. Res. 70(2), 189-196 (2011).

52. R. Hester and H. Garavan, "Working memory and executive function: the influence of content and load on the control of attention," Memory Cognit. 33(2), 221-233 (2005).

53. B. Pearson et al., "Working memory retrieval as a decision process," J. Vision 14, 2 (2014).

54. H. Sato et al., "Intersubject variability of near-infrared spectroscopy signals during sensorimotor cortex activation," J. Biomed. Opt. 10, 044001 (2005).

55. A. K. Singh et al., "Spatial registration of multichannel multi-subject fNIRS data to MNI space without MRI," NeuroImage 27(4), 842-851 (2005).

56. M. Okamoto et al., "Structural atlas-based spatial registration for functional near-infrared spectroscopy enabling inter-study data integration," Clin. Neurophysiol. 120(7), 1320-1328 (2009).

57. Y. Li et al., "A low cost multichannel NIRS spectrometer for monitoring global physiological hemodynamic fluctuations," in Society for Functional Near Infarred Spectroscopy Biennual Meeting, Université Paris Descartes, Paris, France (2016).

58. S. Sutoko et al., "Tutorial on platform for optical topography analysis tools," Neurophotonics 3(1), 010801 (2016).

59. D. T. Delpy et al., "Estimation of optical pathlength through tissue from direct time of flight measurement," Phys. Med. Biol. 33(12), 1433-1442 (1988).

60. K. J. Friston et al., "Analysis of fMRI time-series revisited," NeuroImage 2(1), 45-53 (1995).

61. K. J. Friston et al., "Event-related fMRI: characterizing differential responses," NeuroImage 7(1), 30-40 (1998).

62. K. J. Friston, P. Jezzard, and R. Turner, "Analysis of functional MRI time-series," Hum. Brain Mapp. 1, 153-171 (1994).

63. M. M. Monti, "Statistical analysis of fMRI time series: a critical review of the GLM approach," Front. Hum. Neurosci. 5, 1-13 (2011).

64. P. Pinti et al., "A novel GLM-based method for the automatic identification of functional events (AIDE) in fNIRS data recorded in naturalistic environments," NeuroImage 155, 291-304 (2017).

65. G. H. Glover, T.-Q. Li, and D. Ress, "Image-based method for retrospective correction of physiological motion effects in fMRI: RETROICOR,” Magn. Reson. Med. 44, 162-167 (2000).

66. Y. Tong et al., "Evaluating the effects of systemic low frequency oscillations measured in the periphery on the independent component analysis results of resting state networks," NeuroImage 76, 202-215 (2013).

67. R. J. Cooper et al., "The utility of near-infrared spectroscopy in the regression of low-frequency physiological noise from functional magnetic resonance imaging data," NeuroImage 59(4), 3128-3138 (2012).

68. H. Sato et al., "A NIRS-fMRI investigation of prefrontal cortex activity during a working memory task," NeuroImage 83, 158-173 (2013).

69. F. Tian et al., "Enhanced functional brain imaging by using adaptive filtering and a depth compensation algorithm in diffuse optical tomography," IEEE Trans. Med. Imaging 30(6), 1239-1251 (2011).

70. Y. Hoshi, N. Kobayashi, and M. Tamura, "Interpretation of near-infrared spectroscopy signals: a study with a newly developed perfused rat brain model," J. Appl. Physiol. 90, 1657-1662 (2001).

71. A. Hirasawa et al., "Influence of skin blood flow and source-detector distance on near-infrared spectroscopy-determined cerebral oxygenation in humans," Clin. Physiol. Funct. Imaging 35, 237-244 (2014). 
72. T. Katura et al., "Quantitative evaluation of interrelations between spontaneous low-frequency oscillations in cerebral hemodynamics and systemic cardiovascular dynamics," NeuroImage 31(4), 1592-1600 (2006).

73. G. A. Z. Morais et al., "Non-neuronal evoked and spontaneous hemodynamic changes in the anterior temporal region of the human head may lead to misinterpretations of functional near-infrared spectroscopy signals," Neurophotonics 5(1), 011002 (2018).

74. Y. Zhang et al., "Multiregional functional near-infrared spectroscopy reveals globally symmetrical and frequency-specific patterns of superficial interference," Biomed. Opt. Express 6(8), 2786-2802 (2015).

75. H. Liu et al., "Influence of blood vessels on the measurement of hemoglobin oxygenation as determined by time-resolved reflectance spectroscopy," Med. Phys. 22 (8), 1209-1217 (1995).

76. R. L. van Veen, W. Verkruysse, and H. J. Sterenborg, "Diffuse-reflectance spectroscopy from 500 to $1060 \mathrm{~nm}$ by correction for inhomogeneously distributed absorbers," Opt. Lett. 27(4), 246-248 (2002).

77. O. K. Schwenn et al., "Experimental percutaneous cannulation of the supraorbital arteries: implication for future therapy," Invest. Ophthalmol. Visual Sci. 46(5), 1557-1560 (2005).

78. Y. A. Pinar and F. Govsa, "Anatomy of the superficial temporal artery and its branches: its importance for surgery," Surg. Radiol. Anat. 28(3), 248-253 (2006).

79. M. Uga et al., "Optimizing the general linear model for functional nearinfrared spectroscopy: an adaptive hemodynamic response function approach," Neurophotonics 1(1), 015004 (2014).

80. T. Takahashi et al., "Influence of skin blood flow on near-infrared spectroscopy signals measured on the forehead during a verbal fluency task," NeuroImage 57(3), 991-1002 (2011).

81. I. Tachtsidis et al., "Measurement of frontal lobe functional activation and related systemic effects: a near-infrared spectroscopy investigation," Adv. Exp. Med. Biol. 614, 397-403 (2008).

82. Y. Li et al., "Systemic low-frequency oscillations observed in the periphery of healthy human subjects," J. Biomed. Opt. 23(5), 057001 (2018).

83. C. S. Roy and C. S. Sherrington, "On the regulation of the blood-supply of the brain," J. Physiol. 11(1-2), 85-158 (1890).

84. I. M. Devonshire et al., "Neurovascular coupling is brain regiondependent," NeuroImage 59, 1997-2006 (2012).

85. D. C. Glahn et al., "Maintenance and manipulation in spatial working memory: dissociations in the prefrontal cortex," NeuroImage 17, 201213 (2002).

86. Y. Hoshi et al., "Spatiotemporal characteristics of hemodynamic changes in the human lateral prefrontal cortex during working memory tasks," Neurolmage 20(3), 1493-1504 (2003).

87. S. Tsujimoto et al., "Prefrontal cortical activation associated with working memory in adults and preschool children: an event-related optical topography study," Cereb. Cortex 14(7), 703-712 (2004).

88. E. Takahashi, K. Ohki, and D.-S. Kim, "Dissociation and convergence of the dorsal and ventral visual working memory streams in the human prefrontal cortex," NeuroImage 65, 488-498 (2013).

Stephanie Sutoko received her bachelor's degree in engineering from the Bandung Institute of Technology in 2011 and her master's degree from the University of Tokyo in 2014. She has started her career as a researcher in the Center for Exploratory Research, Research and Development (R\&D) Group, Hitachi, Ltd., Japan, since 2014. Her current research interests include NIRS application in psychiatric, signal processing, and data analysis.

Yee Ling Chan received her BEng (Hons) degrees from the Universiti Teknologi Petronas (UTP) in 2018. She was an intern in the Center for Exploratory Research, Research and Development (R\&D) Group, Hitachi, Ltd., Japan, from 2016 to 2017. She is currently a graduate research assistant in Center for Intelligent Signal and Imaging Research (HICOE-CISIR), UTP. Her primary research focus is on NIRS data analysis and signal processing.

Akiko Obata is a senior researcher in the R\&D Group, Hitachi, Ltd., Japan. Since 2004, she has been a member of the research group working on optical topography and optical brain function monitoring technology based on NIRS. Her main responsibility includes the development of new applications of NIRS.
Hiroki Sato received his master's degree from the University of Tokyo in 2000 and his PhD from Keio University in 2006. He was a senior researcher at R\&D Group, Hitachi, Ltd. from 2001 to 2018 and was a guest researcher at the University of Tuebingen from 2013 to 2014. Currently, he is a professor at Shibaura Institute of Technology, Japan. His research interests include cognitive neuroscience and application of near-infrared spectroscopy.

Atsushi Maki joined Hitachi, Ltd., R\&D Group in 1990. He was an invited researcher of the University College London in 2002. He received his $\mathrm{PhD}$ in medical engineering. He developed the new neuroimaging method named optical topography in 1995. Currently, he engages developmental human science and higher order brain diseases. His research area is the development of new solutions based on brain measurement (e.g., neuropharmacology, diagnosis of brain disease, and applied brain science on industrial area).

Takashi Numata received his $\mathrm{PhD}$ in science from the University of Tokyo in 2015. He has started a career as a researcher in the Center for Exploratory Research, Research and Development (R\&D) Group, Hitachi, Ltd., Japan, since 2015. His current research interests include mental state evaluation by multimoldal biosignal measurement and analysis.

Tsukasa Funane received his bachelor's degree in engineering from the University of Tokyo in 2004 and his PhD in engineering from Keio University in 2012. He is a senior researcher at R\&D Group, Hitachi, Ltd., Japan. He has been a member of the research group working on optical brain function monitoring technology based on NIRS. His research interests include biomedical optics and its neuroscience and medical applications. He is a member of SPIE.

Hirokazu Atsumori is a researcher in the Center for Exploratory Research, R\&D Group, Hitachi, Ltd., Japan. He has been working on the research and development of optical topography - a functional neuroimaging technique since 2002. Currently, he is engaged in the development of multimodality and human-sensing systems for healthcare applications.

Masashi Kiguchi is a senior researcher at the R\&D Group, Hitachi, Ltd., Japan. His background includes physics and laser spectroscopy. $\mathrm{He}$ is interested in problems related to the principle of NIRS measurement. He has been taking the lead in the development of new techniques for observing brain activities to open new research fields and in basic studies for putting them to practical use.

Tong Boon Tang received his $\mathrm{PhD}$ and BEng (Hons) degrees from the University of Edinburgh. Currently, he is the director at the Institute of Health and Analytics for Personalized Care, UTP, Malaysia. His research interests are in biomedical instrumentation, from device and measurement to data fusion. He serves as an editorial board member of the Journal of Medical Imaging and Health Informatics and a vice chair of the IEEE Circuits and Systems Society Malaysia Chapter.

Yingwei Li is a professor at the School of Information Science and Engineering at Yanshan University, China. He received his MSc and $\mathrm{PhD}$ degrees in circuits and systems at Yanshan University in 2003 and 2008, respectively. He has been working in fields related to sensing technology and instruments since 2000 . Now his research focuses on NIRS imaging, THz stimulation, and ultrasonic detection.

Blaise deB Frederick is an associate professor of psychiatry at Harvard Medical School. He studied physics at Yale University and received his $\mathrm{PhD}$ in biophysics from the University of California at Berkeley in 1994. His primary research focus is using the interaction between systemic physiological noise processes and fMRI and fNIRS data to elucidate hemodynamic changes in neuropsychiatric and circulatory disorders.

Yunjie Tong is an assistant professor at Weldon School of Biomedical Engineering at Purdue University. He studied physics at Peking University, China, and received his $\mathrm{PhD}$ in biomedical engineering from Tufts University in 2008. His research interests are the developments of new analytical and experimental methods in fMRI and fNIRS to remove physiological noise and deepen our understanding of brain function and perfusion. 